\title{
Characterizing the genetic basis of copper toxicity in Drosophila reveals a complex pattern of allelic, regulatory, and behavioral variation
}

\author{
Elizabeth R. Everman (D) , ${ }^{1, *}$ Kristen M. Cloud-Richardson, ${ }^{1}$ and Stuart J. Macdonald (D) 1,2 \\ ${ }^{1}$ Department of Molecular Biosciences, University of Kansas, Lawrence, KS 66045, USA \\ ${ }^{2}$ Center for Computational Biology, University of Kansas, Lawrence, KS 66047, USA \\ ${ }^{*}$ Corresponding author: Department of Molecular Biosciences, 4043 Haworth Hall, 1200 Sunnyside Avenue, University of Kansas, Lawrence, KS 66045, USA. \\ e.everman@ku.edu
}

\begin{abstract}
A range of heavy metals are required for normal cell function and homeostasis. However, the anthropogenic release of metal compounds into soil and water sources presents a pervasive health threat. Copper is one of many heavy metals that negatively impacts diverse organisms at a global scale. Using a combination of quantitative trait locus (QTL) mapping and RNA sequencing in the Drosophila Synthetic Population Resource, we demonstrate that resistance to the toxic effects of ingested copper in D. melanogaster is genetically complex and influenced by allelic and expression variation at multiple loci. QTL mapping identified several QTL that account for a substantial fraction of heritability. Additionally, we find that copper resistance is impacted by variation in behavioral avoidance of copper and may be subject to life-stage specific regulation. Gene expression analysis further demonstrated that resistant and sensitive strains are characterized by unique expression patterns. Several of the candidate genes identified via QTL mapping and RNAseq have known copper-specific functions (e.g., Ccs, Sod3, CG11825), and others are involved in the regulation of other heavy metals (e.g., Catsup, whd). We validated several of these candidate genes with RNAi suggesting they contribute to variation in adult copper resistance. Our study illuminates the interconnected roles that allelic and expression variation, organism life stage, and behavior play in copper resistance, allowing a deeper understanding of the diverse mechanisms through which metal pollution can negatively impact organisms.
\end{abstract}

Keywords: heavy metal resistance; copper; life stage-specific; DSPR; QTL; MPP

\section{Introduction}

Anthropogenic release of heavy metals into soil and water sources presents a pervasive threat with long-lasting ecological, health, and economic impacts (Wu et al. 1975; Babin-Fenske and Anand 2011; Wuana and Okieimen 2011; Gall et al. 2015). Elevated heavy metals have been reported in dozens of organisms at all levels of the ecosystem (Neuberger et al. 1990; Georgieva et al. 2002; Sánchez-Chardi and Nadal 2007; Usmani 2011; Gall et al. 2015; Wright et al. 2015; Ecke et al. 2017; Plessl et al. 2017; Ilunga Kabeya et al. 2018), demonstrating that heavy metal pollution is wide reaching and can spread through food webs (Gall et al. 2015; Ilunga Kabeya et al. 2018). Although required for normal physiological function at low concentrations, copper is one of many common environmental heavy metal pollutants linked to mining (Ramirez et al. 2005; Wuana and Okieimen 2011; Wright et al. 2015), drinking water pipes (Harvey et al. 2016), and pesticide and fertilizer use (de Oliveira-Filho et al. 2004; Wuana and Okieimen 2011). In its essential role, copper helps bind oxygen, catalyzes enzymatic reactions, and promotes normal neurological development (Hart et al. 1928; Danks 1988; World Health Organization et al. 1996; Uriu-Adams and Keen 2005; Norgate et al. 2006;
Navarro and Schneuwly 2017). However, excessive copper exposure ultimately leads to the overproduction of reactive oxygen species (ROS), which can cause cellular damage through oxidative stress (Uriu-Adams and Keen 2005; Tchounwou et al. 2008).

Evolutionarily conserved metal-responsive transcription factor 1 (MTF-1) and metallothionein (MT) proteins function as a first line of defense against toxic effects of excessive copper exposure in diverse organisms including humans, flies, fungi, and plants (Macnair 1993; Goldsbrough 2000; Bellion et al. 2007; Calap-Quintana et al. 2017). MTF-1 binds to metal responsive elements of MT genes, increasing MT abundance in copper accumulating cells and allowing excess heavy metal ions to be sequestered until they are removed from the system (Filshie et al. 1971; Stuart et al. 1985; Mokdad et al. 1987; Egli et al. 2003; Balamurugan et al. 2004; Southon et al. 2004). Metal chaperone and transporter proteins such as Ccs (Culotta et al. 1997), Atox1 (Southon et al. 2004; Hatori and Lutsenko 2013), ATP7 (Norgate et al. 2006), and CTR1 family transporters (Petris et al. 2003; Guo et al. 2004; Balamurugan et al. 2007; Turski and Thiele 2007; Calap-Quintana et al. 2017; Navarro and Schneuwly 2017) also play a crucial role in the response to heavy metal toxicity (Egli et al. 2003; Petris et al. 2003; Yepiskoposyan et al. 2006; Janssens 
et al. 2009). For example, in Drosophila melanogaster, high copper exposure decreases translation of Ctr1A and Ctr1B via MTF-1 to reduce influx of copper ions (Balamurugan et al. 2007; Turski and Thiele 2007; Calap-Quintana et al. 2017; Navarro and Schneuwly 2017), whereas high copper exposure in humans leads to degradation of the hCTR1 protein (the human ortholog of Ctr1A/B) and a reduction in the intracellular concentration of copper (Petris et al. 2003; Guo et al. 2004).

Much of our understanding of the response to copper stress has come from studies that use genetic manipulation to define the roles of metal responsive genes (e.g., Egli et al. 2003, 2006a; Bellion et al. 2007; Kirby et al. 2008; Bahadorani et al. 2010). However, quantitative trait locus (QTL) and GWA (genome-wide association) studies have demonstrated the genetic complexity of the response to heavy metal stress (Courbot et al. 2007; Willems et al. 2007; Zhou et al. 2017). For example, QTL mapping using Caenorhabditis elegans recombinant inbred advanced intercross lines showed that several regions of the genome are involved in the response to cadmium, copper, and silver exposure (Evans et al. 2018). GWA with the Drosophila Genetic Reference Panel (DGRP) revealed multiple candidate loci associated with the response to cadmium and lead stress (Zhou et al. 2017). QTL mapping of metal resistance in plants has further demonstrated the role that allelic variation plays in the response to heavy metal stress (Courbot et al. 2007; Willems et al. 2007; Turner et al. 2010; Arnold et al. 2016). For instance, an interspecific QTL study of two closely related species of Arabidopsis (metal-tolerant A. haller$i \times$ metal-sensitive A. lyrata petraea) identified multiple regions of the genome that contributed to zinc and cadmium resistance, and demonstrated that metal resistant alleles had become fixed in the metal tolerant species in the populations sampled (Courbot et al. 2007; Willems et al. 2007). Similarly, sequencing of A. arenosa populations locally adapted to serpentine soils revealed strong selection for introgressed alleles from the more tolerant A. lyrata (Arnold et al. 2016). These and other examples from Mimulus gutattus growing in copper mine tailings (Allen 1971; Macnair 1983; MacNair et al. 1993; Wright et al. 2015; Selby and Willis 2018) highlight the utility of using a quantitative genomics approach with powerful mapping panels to examine the influence of allelic variation on metal tolerance.

Drosophila melanogaster is an important model for understanding the mechanisms involved in the response to toxic heavy metal exposure due to the ease with which it can be genetically manipulated (e.g., Egli et al. 2006a; reviewed in Navarro and Schneuwly 2017) and because of the extensive conservation of genes involved in the response to metal ions between flies and humans (Calap-Quintana et al. 2017). In addition, the existence of large Drosophila mapping panels makes this model system especially well-suited for examining the effect of naturally occurring alleles on the response to heavy metal stress. Finally, since D. melanogaster is a widespread species that is closely associated with human activities, and is commonly found in agricultural settings (Keller 2007; e.g., Gleason et al. 2019) where heavy metalcontaining pesticides, fungicides, and fertilizers may be in use, understanding the response to heavy metal exposure in flies has potential ecological and agricultural relevance.

In this study, we used more than 1500 strains from the Drosophila Synthetic Population Resource (DSPR) (King et al. 2012a, $2012 b)$ to investigate the influence of allelic variation on the response to toxic copper exposure through QTL mapping, used RNA sequencing of copper-resistant and copper-sensitive strains to assess changes in gene expression following copper exposure, and followed up several plausible candidate genes with RNAi.
Since a number of genes are known to respond to multiple heavy metals (Calap-Quintana et al. 2017), and pleiotropic QTL can underlie genetic variation for multiple metal resistance traits (Evans et al. 2018), our findings on the genetic architecture of copper resistance have the potential to provide broader insight into the allelic and expression variation influencing heavy metal stress.

\section{Materials and methods Mapping panel}

We reared and phenotyped the $>1500$ recombinant inbred lines (RILs) that comprise the DSPR to measure variation in susceptibility to copper stress. The DSPR is a multiparental, advanced generation intercross mapping panel derived from 15 unique and fully sequenced founder strains, which represent a global sampling of genetic diversity in D. melanogaster. The DSPR consists of two mapping panels ( $A$ and $B$ ), which are composed of two subpanels (A1 and A2, and B1 and B2). The subpanels were started from the same set of founders, but were maintained independently [see King et al. (2012b) for additional details on the mapping panel].

\section{Rearing and assay conditions}

Strains from the DSPR were maintained, reared, and tested in the same incubator under a $12: 12 \mathrm{~h}$ light:dark photoperiod at $25^{\circ} \mathrm{C}$ and $50 \%$ humidity. To obtain female flies for the adult copper resistance assay, RNA sequencing, and RNAi validation, adults were transferred to cornmeal-molasses-yeast food, allowed to oviposit for two days, then discarded. Experimental female, presumably mated, flies from the following generation were sorted over $\mathrm{CO}_{2}$ and placed into vials with new cornmeal-molassesyeast media for $24 \mathrm{~h}$ before they were transferred to coppersupplemented food. All adult assays were performed on 3- to 5day-old individuals.

\section{Adult female copper resistance}

The adult female response to copper stress was measured as percent survival on media containing $50 \mathrm{mM} \mathrm{CuSO}_{4}$ following a 48-h exposure period. As essentially no flies die on control food (Highfill et al. 2016) or under starvation conditions (Everman et al. 2019) throughout the span of our assay, we did not assess adult female survival on control food in this study. Experimental females were transferred without $\mathrm{CO}_{2}$ anesthesia to vials containing $1.8 \mathrm{~g}$ potato-based Instant Drosophila Medium (Carolina Biological Supply Company 173200) hydrated with $8 \mathrm{~mL} 50 \mathrm{mM}$ $\mathrm{CuSO}_{4}$ [Copper(II) sulfate, Sigma-Aldrich C1297]. Instant Drosophila Medium is estimated to contain approximately $0.02 \mathrm{mM}$ Cu prior to hydration (Maroni and Watson 1985). Copper resistance was measured in a total of 11 batches across the $A(N$ strains $=767)$ and B (N strains $=789)$ DSPR mapping panels. Each strain was measured in a single batch with three vial replicates each containing between 7 and 20 individuals (average number of flies per vial replicate $=19.4$ ). The effect of copper on survival was reported as mean percent survival per strain across the three replicate vials. Retaining vials with fewer than 15 flies did not impact our QTL mapping results in a meaningful way (see below). Hereafter, the adult survival response to $48 \mathrm{~h}, 50 \mathrm{mM} \mathrm{CuSO}_{4}$ is referred to as adult copper resistance.

\section{Adult female feeding response to copper- supplemented media}

A subset of strains evenly sampled throughout the B2 subpanel adult copper resistance distribution (0\% \pm 0 S.E. $-98.4 \% \pm 1.59$ S.E.) were used to measure the effect of copper exposure on food 
intake. We measured food intake in three blocks with at least two vial replicates of 20 females per strain $(N=95)$ per treatment (control us copper) with vial replicates distributed across blocks, following a protocol modified from (Shell et al. 2018). Briefly, we added 1\% erioglaucine disodium salt (Sigma-Aldrich 861146), a blue dye, to water and to $50 \mathrm{mM} \mathrm{CuSO}_{4}$ no more than $24 \mathrm{~h}$ prior to the assay to avoid dye decomposition. We hydrated $0.9 \mathrm{~g}$ Instant Drosophila Medium with $4 \mathrm{~mL}$ liquid, and flies were allowed to consume dyed food for $24 \mathrm{~h}$ before they were frozen for up to $5 \mathrm{~h}$. No flies died during the 24-h period. Subsequently, flies were homogenized with three to four glass beads in $600 \mu \mathrm{L}$ distilled water for $45 \mathrm{~s}$ using a Mini-Beadbeater-96 (BioSpec Products). Homogenate was centrifuged for $5 \mathrm{~min}$ at $14,000 \mathrm{rpm}$, and $200 \mu \mathrm{L}$ supernatant was transferred to a 96 -well plate. Fly homogenate was frozen for up to $48 \mathrm{~h}$ before absorbance at $630 \mathrm{~nm}$ was measured with a BioTek Multimode Microplate reader (Synergy 2 v.1.03). Two water blanks and 14 standards ranging from $6.25 \times 10^{-5} \%$ to $0.006 \%$ dye in water were prepared fresh for each block and were included in each plate to determine the dye concentration of fly homogenate, and to assess consistency among blocks. Absorbance readings for standards were highly correlated across plates and blocks (Supplementary Table S1). To calculate the estimated amount of dye consumed, we used a linear model to find the slope and intercept of the standard curve (Concentration of Standard $\sim$ Absorbance $\times$ Block). Estimated percent dye in each fly homogenate sample corrected for block variation observed among standards was determined with the equation:

$$
\% \text { Dye in sample }=(0.002443 \times \text { absorbance })-0.0001465 .
$$

Variation in feeding behavior among DSPR strains on copper and control food was assessed with a two-way ANOVA with an interaction (\% Dye Consumed $\sim$ DSPR Strain $\times$ Treatment), and effect size was calculated using Cohen's F ( $R$ package sjstats) (Cohen 1988; Lüdecke 2018). The correlations between feeding behavior (average percent dye consumed per RIL) on copper and control food and adult copper resistance were assessed with linear models (\% Dye Consumed Adult Copper Resistance). Feeding plasticity was calculated as the percent dye consumed on control food minus the percent dye consumed on coppersupplemented food, and the correlation between feeding plasticity and adult copper resistance was tested with a linear model (Feeding Plasticity Adult Copper Resistance).

\section{Developmental response to copper}

Developmental viability was estimated in the B panel from 100 strains that were evenly sampled from throughout the distribution of B1 and B2 subpanel adult copper resistance $(0 \% \pm 0$ S.E. $-98.4 \% \pm 1.59$ S.E.). Approximately 100 females per strain were allowed to oviposit on cornmeal-molasses-yeast media for $17-20 \mathrm{~h}$ in $6 \mathrm{oz}$ polypropylene Drosophila bottles (Genesee Scientific: 32-130) with yeast paste to encourage egg laying. Following oviposition, remaining yeast paste was removed, and embryos were gently dislodged from the media surface by rinsing with $1 \times$ PBS and swirling with a small, bristled paintbrush. Subsequently, for each strain, we arrayed multiple $10 \mu \mathrm{L}$ aliquots of embryos suspended in $1 \times$ PBS onto a petri dish containing $2 \%$ agar dyed blue with Erioglaucine Disodium Salt (Sigma-Aldrich 861146; $8 \mathrm{mg} / \mathrm{mL}$ ). For each dish, we aliquoted eggs into 14 cells (Supplementary Figure S1), photographed the dish (Nikon D3200, $105 \mathrm{~mm}$ 1:2.8 DG Sigma Macro lens), and the number of embryos within each cell was recorded with Imagej (v. 1.51s). Embryos from each cell (30-306 embryos, average $=125$ embryos) were then transferred with a rubber, bristleless paintbrush to vials containing control or $2 \mathrm{~mm} \mathrm{CuSO}_{4}$ hydrated Instant Drosophila Medium (1.8g media plus $8 \mathrm{~mL}$ liquid). The rubber paintbrush was examined after each egg transfer to ensure all eggs had been transferred to the vial. The developmental response to copper was assessed with 4-7 replicates per treatment for each strain (mean replicates per strain $=6.8$ ). We used a lower copper concentration in this assay because previous studies have shown that the larval life stage is much more susceptible to copper toxicity compared to adults (Bahadorani and Hilliker 2009).

Copper stress has the potential to increase development time and reduce the number of individuals that complete development from egg to adult. To estimate the effect of copper exposure on development time, for each experimental vial, we recorded the number of days between set up and the first emergence of adults. We acknowledge this measure limits our assessment of the distribution of adult emergence times per vial, but we found that removing adult flies throughout the assay disrupted developing pupae, potentially impacting subsequent emergence counts. To assess the effect of copper on developmental viability, we calculated the proportion of embryos in each vial that eclosed as adults in the seven days following the day of first emergence. Developmental viability was square root transformed to improve deviation from normality within treatment (Shapiro-Wilks test on transformed data; $W=0.99, P=0.04$ ). From here forward, square-root transformed developmental viability is simply referred to as developmental viability, and all subsequent analyses were performed on square-root transformed data. Vials were monitored daily for 30 days after set up. Of the 1356 vials included in this assay, 100 copper treatment vials yielded zero flies. These vials were given a development time of 30 days.

We used a two-way ANOVA with an interaction to measure the effect of strain and treatment on each developmental trait. The DSPR strains we used in this study varied in development time $\left(F_{99,579}=11.8, \quad P<0.00001 ; \quad\right.$ Supplementary Table S2A) and developmental viability on control food $\left(F_{(99,579)}=31.6\right.$, $\mathrm{P}<0.00001$; Supplementary Table S2B). Furthermore, regression analysis demonstrated that development time and developmental viability in control and copper conditions were correlated (development time: $\quad F_{(1,98)}=61.0, \quad P<0.0001, \quad R^{2}=38 \%$; Supplementary Table S2C and Figure S2A; developmental viability: $F_{(1,98)}=54.1, P<0.0001, R^{2}=36 \%$; Supplementary Table S2D and Figure S2B). Therefore, we employed linear models to regress out variation in control development time and control developmental viability to more directly assess the effect of copper stress on these metrics of development. Residual development time and residual developmental viability are referred to from hereafter as treatment-specific development time and treatment-specific developmental viability, respectively.

\section{Heritability}

We estimated the genetic and phenotypic variances of adult copper resistance, control and copper feeding responses, treatmentspecific development time, and treatment-specific developmental viability using linear mixed models (lme and varcomp functions in R; R package: APE, Paradis et al. 2004; R package: nlme, Pinheiro et al. 2017). For all responses, panel-specific broad-sense heritabilities were calculated as the proportion of the total strainspecific variation explained by the estimated genetic variance component (Lynch and Walsh 1998). 


\section{QTL mapping of life stage-specific response to copper stress}

We used the DSPRqtl package in R (github.com/egking/DSPRqtl; FlyRILs.org) to identify QTL associated with variation in all measured traits. QTL mapping was performed for each mapping panel (A and B) and phenotype separately. At each position in the genome, for each strain, we can estimate the additive probability that the segment of the genome was inherited from each of the eight DSPR founders. QTL were identified by regressing the strain mean phenotype on these probabilities, and significance thresholds were assigned following 1000 permutations of the data (King et al. 2012a, 2012b). For adult copper resistance, peak positions for each QTL were estimated with 2-LOD support intervals (King et al. 2012a). Because fewer strains were used to measure the feeding and treatment-specific development traits, to better approximate a 95\% confidence interval on QTL position, we used a 3-LOD drop (King et al. 2012a). Using a 3-LOD drop inevitably has the effect of broadening the QTL interval, which may increase the likelihood of detecting overlap among QTL mapped for different traits, and lead to a spurious inference of pleiotropy. However, we found no difference in the number of QTL that overlapped among traits when employing 2- or 3-LOD drops, and therefore only present results based on 3-LOD drops below. We performed gene ontology (GO) analysis without normalizing for gene length for genes included in peak intervals for each trait and mapping panel separately (FlyMine.org; Lyne et al. 2007), but saw no GO enrichment likely because QTL intervals include many non-causative genes that potentially obscure any signal of enrichment.

Adult copper resistance varied between the A1 and A2 subpanels but did not vary between the B subpanels (A panel: $F_{1,2289}=12.64 ; \quad P<0.001 ; \quad B \quad$ panel: $\quad F_{1,2495}=0.03 ; \quad P=0.86$; Supplementary Figure S3 and Table S2, E-F). Therefore, subpanel was included as a model covariate only in the QTL analysis of panel A. Phenotyping batch also significantly influenced variation in adult copper resistance in both the A and B panels (Supplementary Table S2, E-F). Because batch and subpanel were confounded, we could not test the effects of both covariates on adult copper resistance at the same time. However, including batch as a covariate in the QTL mapping model did not drastically alter the estimation of LOD scores for either panel (A panel correlation between LOD scores $=99 \%$; B panel correlation $=98 \%$; Supplementary Figure S4A) or the presence of any QTL, so we only present data from the models lacking batch as a covariate. Because the development assay was conducted on 100 strains across 15 batches, DSPR strain was highly confounded with batch. Therefore, we did not include batch or subpanel as a covariate in the QTL mapping models for treatment-specific development time or treatment-specific developmental viability. As each strain assessed in the feeding response assay was measured in each of three blocks, block could not be included in the model for either average feeding response on control or copper food.

To determine whether including vials containing relatively few flies influenced QTL mapping results due to mis-estimated phenotype means, we additionally mapped variation in adult copper resistance using only data from vials containing at least 15 flies (removing 316 or $7 \%$ of the vials). LOD scores for the full data set were highly correlated with those for the reduced dataset for each panel (A panel correlation=99\%; B panel correlation $=99 \%$; Supplementary Figure S4B) and the same QTL were identified in each analysis, so we only present data from analyses using all vials.

\section{QTL-centered association tests}

Since all DSPR founders are sequenced, we can use the founder haplotype structure of each RIL to infer the allele that each RIL possesses at each variant segregating among the founders. We can then fit a single marker model at each variant beneath a mapped QTL to associate phenotype and genotype, and examine whether individual variant sites can explain QTL peaks (see Kislukhin et al. 2013; King et al. 2014; Marriage et al. 2014 for more detail). In principle, executing such analyses can help resolve, or fine-map QTL identified in multiparental mapping populations (see for instance Figure 5 from Gatti et al. 2014).

\section{Differential gene expression in high and low adult copper resistance strains}

We examined gene expression variation in a subset of 10 strains (six with high adult resistance: 76-98\% survival, and four with low adult resistance: $0.0-18 \%$ survival) from the B panel to explore how adult copper resistance class and gene expression interact when individuals are exposed to $50 \mathrm{mM} \mathrm{CuSO}_{4}$. Twenty experimental females from each DSPR strain were transferred to Instant Drosophila Medium hydrated with either water as a control or $50 \mathrm{mM} \mathrm{CuSO}_{4}$ (the same concentration used for the adult resistance assay) for $9 \mathrm{~h}$. No individuals died during the 9 -h exposure period. Following exposure, 10 females from each strain and treatment were flash frozen in liquid nitrogen, placed in TRIzol Reagent (Invitrogen, 15596018), and immediately stored at $-80^{\circ} \mathrm{C}$. RNA was extracted from each of the 20 samples with the Directzol RNA Miniprep kit (Zymo Research, R2050), eluted in 100- $\mu \mathrm{L}$ water, and stored at $-80^{\circ} \mathrm{C}$. We prepared libraries with the TruSeq Stranded mRNA kit (Illumina, 20020595), and paired-end 37-bp mRNA libraries were each sequenced to $\sim 20$ million reads on an Illumina NextSeq 550 at the University of Kansas Genome Sequencing Core.

Sequence quality assessment and trimming were performed using fastp (Chen et al. 2018). We used kallisto to perform pseudoalignment-based mapping of reads (Ensembl transcriptome release 90) (Bray et al. 2016), and performed differential expression analysis with sleuth (v.0.30.0) using likelihood ratio tests (Pimentel et al. 2017). Gene expression is likely to vary between the different DSPR strains; however, we were primarily interested in understanding whether there are consistent differences in gene expression between high and low resistance classes of strains. Given this interest, we treated each strain as a biological replicate of the high and low resistance classes and did not include DSPR strain in differential expression models. After determining that the interaction between resistance class and treatment did not influence expression of any gene at a 5\% FDR (False Discovery Rate), we tested the additive effects of resistance class and treatment on gene expression, referred to from here as the full model (full model: TRT + RES us reduced model: $\sim 1$ ). We also examined the influence of each term independently in two additional models. The effect of treatment alone was assessed by accounting for resistance class (treatment model: $\sim$ TRT + RES Us reduced model: $\sim$ RES), and the effect of resistance class alone was assessed by accounting for treatment (resistance model: $\sim$ TRT + RES us reduced model: $\sim$ TRT). From here on, these termspecific models are referred to as the treatment model and the resistance model, respectively. Significantly differentially expressed (DE) genes for each model were identified with a 5\% FDR threshold.

We generated three lists of significantly DE genes: full model DE genes, treatment model DE genes, and resistance model DE 
genes. Sleuth applies a filter against genes with low expression (Pimentel et al. 2017). We applied an additional filter following sleuth analysis to remove genes from DE gene lists with average expression of less than 1 TPM (Transcripts Per Million). Additionally, we eliminated genes with expression variance $\geq 1$ TPM in any of the following four categories: sensitive strains, control treatment; sensitive strains, copper treatment; resistant strains, control treatment; resistant strains, copper treatment (Supplementary Figure S5). We used principal components analysis (PCA) to examine the effect of treatment and resistance class using quantile normalized TPM data for DE genes. DE gene lists were examined for co-regulated clusters of genes using Clust (Abu-Jamous and Kelly 2018). GO analysis was performed for each cluster and for each of the DE gene lists in their entirety (FlyMine.org; Lyne et al. 2007).

\section{RNAi knockdown of candidate genes associated with adult copper resistance}

Several candidate genes were implicated by QTL mapping and/or RNAseq analysis of adult females. TRiP UAS-RNAi strains (Perkins et al. 2015) for candidate genes, as well as a control UASLUC.VALIUM10 strain, were obtained from the Bloomington Drosophila Stock Center (BDSC) (Supplementary Table S3). Crosses involved 10 TRiP males and 10 virgin females from Gal4expressing driver strains. Each TRiP strain was crossed to both a ubiquitous Gal4 driver strain (BDSC 25374), with Gal4 under the control of the Act5C promoter, and a strain expressing Gal4 in the adult anterior midgut (1099 from Nicholas Buchon, flygut.epfl.ch; Buchon et al. 2013). Three candidate gene TRiP strains (swm, Catsup, and CG11825) produced too few flies to test when crossed to the ubiquitous Gal4-expressing driver and were thus excluded from our analysis. We tested two TRiP UAS RNAi strains for the genes CG5235, MtnC, and ZnT41F to assess the consistency in the effect of gene knockdown on copper survival (Supplementary Table S3).

An average of 19.3 Gal4-UAS RNAi females (min 7) were transferred to Instant Drosophila Medium hydrated with $50 \mathrm{mM} \mathrm{CuSO}_{4}$ using an average of 16.8 ( $\mathrm{min} 10)$ replicate vials per genotype (a total of 203-365 individuals per genotype) across four batches. We counted flies daily until all were dead, and the response to copper stress in these RNAi knockdown genotypes was quantified as average lifespan. We chose to measure lifespan on copper instead of percent survival at $48 \mathrm{~h}$ (as in our DSPR screen) because we had no a priori expectation that survival would be variable at $48 \mathrm{~h}$ among the RNAi genotypes, and knockdown in genes hypothesized to influence the response to copper toxicity could drastically reduce or extend survival. To establish that GAL4UAS-RNAi genotypes were not inherently unhealthy, we additionally placed 20 such females from each cross on Instant Drosophila Medium hydrated with water to assess overall viability. No individuals died on copper-free media through the duration of the RNAi assay. We compared copper resistance for each RNAi knockdown to its respective control using per vial average lifespan controlling for batch with a two-way ANOVA (Average Lifespan $\sim$ Strain $\times$ Batch) with planned comparisons. These analyses were performed separately for each GAL4 driver.

\section{Data availability}

All raw data and images generated from this study, including adult and developmental copper resistance traits, feeding data, raw QTL mapping data, normalized TPM expression data, and RNAi data are available at FigShare. Supplementary File S1 contains descriptions for all accompanying data files. DSPR genotype information is publicly available at www. FlyRILs.org. RNAseq reads are available from NCBI SRA PRJNA633166. Unless otherwise stated, all analyses were performed in $R$ (v. 3.6.2) ( $R$ Core Team 2017).

Supplemental material is available at figshare DOI: https:// doi.org/10.25386/genetics.13283981

\section{Results \\ Abundant variation in adult female copper resistance}

We measured adult copper resistance in females from over 1500 DSPR RILs by exposing 60 flies (3 vials of 20 flies) from each strain to $50 \mathrm{mM} \mathrm{CuSO}_{4}$ for $48 \mathrm{~h}$. Phenotypic variation and heritability were high for female copper resistance in both the A and B panels of the DSPR (A panel: $\mathrm{H}^{2}=83.0 \%$; B panel: $\mathrm{H}^{2}=78.8 \%$; Figure 1).

\section{Adult female feeding response to copper- supplemented media}

Since the toxicity of copper in our assay likely stems from ingestion, we were interested in flies' feeding response to coppersupplemented media. Using a sample of 95 strains from the B2 subpanel that spanned the distribution of adult copper resistance (from 0\% to 98.4\% survival), we tested the effect of $50 \mathrm{mM} \mathrm{CuSO}_{4}$ on feeding behavior. We estimated feeding by measuring the amount of dye consumed by flies exposed to food hydrated with water or a copper solution within a 24 -h period. Both DSPR strain and treatment significantly influenced feeding (DSPR Strain: $F_{94,384}=3.08, \quad P<0.00001$; Treatment: $F_{1,384}=2306, \quad P<0.00001$, Supplementary Table S2G; Figure 2A), although treatment had a much larger effect on the feeding response than strain (Cohen's F: Treatment $=2.57$, DSPR strain $=0.91$ ). We also observed an interaction between strain and treatment (DSPR Strain $\times$ Treatment: $F_{94,384}=2.77, P<0.00001$, Cohen's $F=0.86$, Supplementary Table $\mathrm{S} 2 \mathrm{G}$ ), indicating that the reduction in feeding due to copper is not uniform across strains (Figure 2A). Both feeding responses had high heritability (control feeding response: $\mathrm{H}^{2}=87.6 \%$; copper feeding response: $\mathrm{H}^{2}=87.6 \%$ ).

Overall, feeding behavior under copper conditions was negatively correlated with adult copper resistance $(R=-34.6 \%$, $F_{1,93}=12.7, \quad P=0.0006$; Supplementary Table $S 2 H$, Figure $2 B$ ), while feeding behavior under control conditions was not correlated with adult copper resistance $\left(R=10.2 \%, F_{1,93}=0.98, P=0.32\right.$; Supplementary Table S2I, Figure 2B). Feeding plasticity (calculated as the difference in percent dye consumed on control and
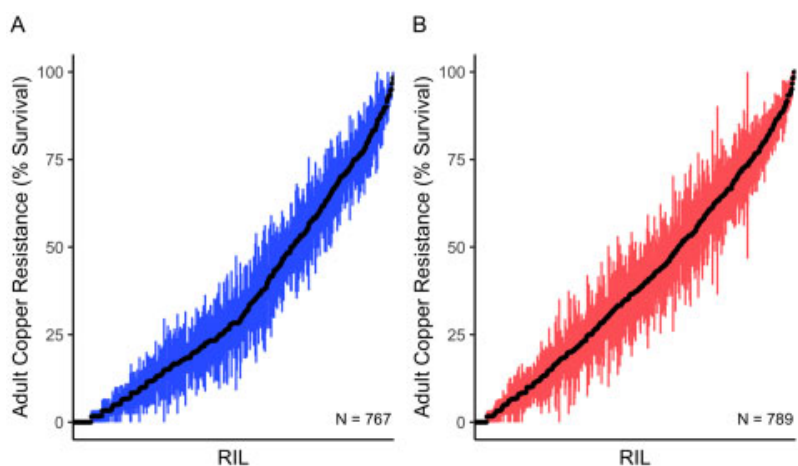

Figure 1 Adult copper resistance is highly variable in the DSPR. Variation in mean female adult copper resistance ( \pm S.E.) per DSPR strain in (A) and (B) panels following 48-h exposure to $50 \mathrm{mM} \mathrm{CuSO}_{4}$. Recombinant inbred lines (RILs) are ordered by phenotype along the $\mathrm{x}$ axis. 
A

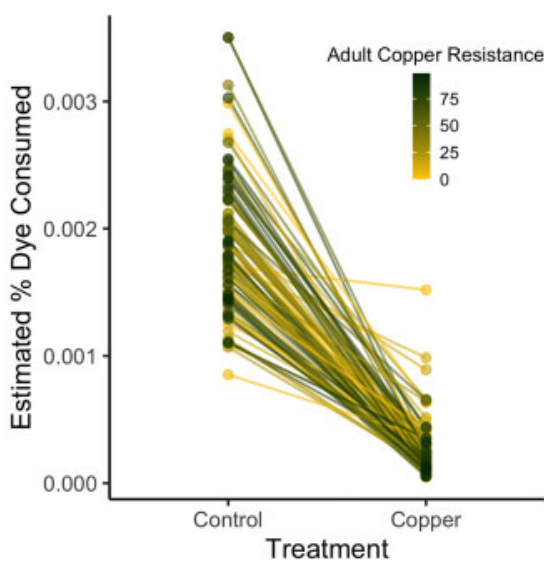

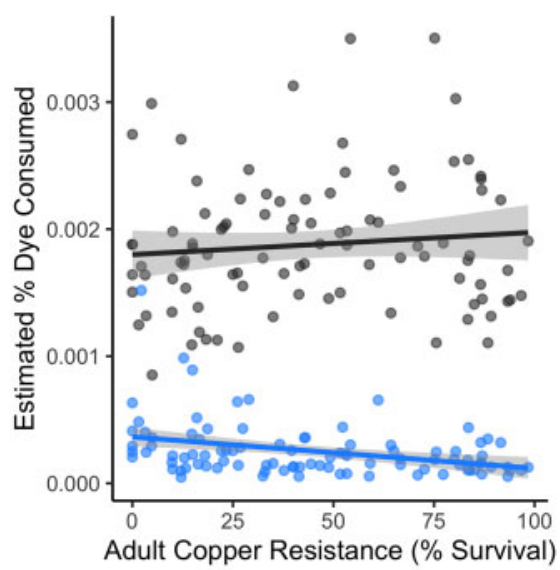

C

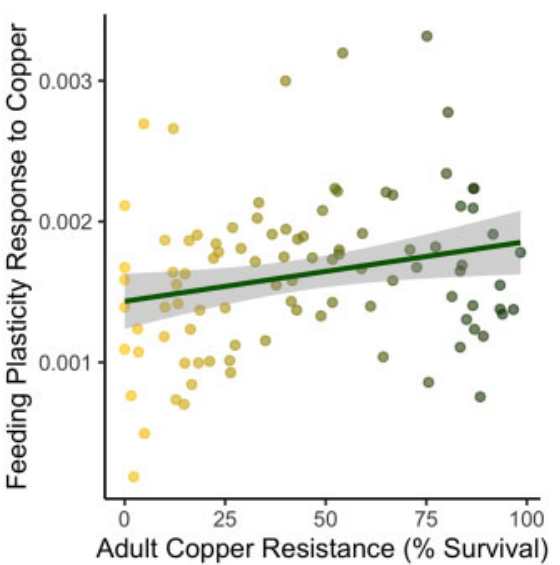

Figure 2 Feeding aversion to copper varies in the DSPR. Feeding behavior in 95 DSPR strains changed in response to 24-h exposure to $50 \mathrm{mM}^{\mathrm{C}} \mathrm{CuSO}{ }_{4}$. (A) Mean percent dye consumed varied among DSPR strains $(P<0.00001)$ and was much lower under copper conditions relative to control (water) conditions (Treatment: $P<0.00001)$. The interaction between strain and treatment $(P<0.00001)$ suggests that the feeding response to copper varies among the strains. (B) Feeding behavior under control conditions was not correlated with adult copper resistance $(P=0.32)$; feeding behavior on copper was correlated with adult copper resistance $(P=0.0006)$. Feeding response to copper is shown in blue; the control response is shown in black. ( $C$ ) Feeding plasticity (calculated as the difference in dye consumption in control and copper food) was correlated with adult copper resistance ( $P=0.02)$, suggesting that resistant strains displayed greater behavioral plasticity in response to copper compared to sensitive strains. Point color indicates adult copper resistance, as in (A). Shading around the regression in (B) and (C) indicates the $95 \% \mathrm{CI}$ of the regression.

copper media) was weakly positively correlated with adult copper resistance $\left(F_{1,93}=5.33, P=0.02, R^{2}=0.5 \%\right.$; Supplementary Table $\mathrm{S} 2 \mathrm{~J})$, suggesting that copper resistant strains display greater behavioral plasticity in response to copper-supplemented food compared to sensitive strains (Figure 2C). Together, these results suggest that our adult copper resistance phenotype is partially influenced by a copper-induced behavior, where more sensitive strains tend to eat more copper food than more resistant strains in a 24-h period. Equally, the limited strength of the relationship likely implies our resistance phenotype is primarily impacted by the physiological and metabolic response to copper and is not solely influenced by behavioral avoidance.

\section{Developmental response to copper}

In organisms with complex life cycles, the genetic control of physiological traits can be decoupled between life stages (Freda et al. 2017; Collet and Fellous 2019). To assess whether the strains with high resistance to copper as adults were also more resistant in other life stages, we sampled 100 strains from the B1 and B2 subpanels that span the range of adult copper resistance (from 0\% to $98.4 \%$ survival). Embryos from these strains were placed on control media or media containing $2 \mathrm{mM} \mathrm{CuSO}_{4}$, and the day of first adult emergence (development time) and the proportion of embryos that emerged as adults (developmental viability) were recorded. Both development time and developmental viability were variable among strains on copper and control media (development time: $F_{99,1157}=24.21, P<0.00001$; developmental viability: $F_{99,1157}=49.17 .21, \quad P<0.00001$; Supplementary Table S2, K-L, Figure 3). Exposure to copper delayed emergence by nearly 4 days on average $\left(F_{1,1157}=1293, P<0.00001\right.$; Supplementary Table S2K, Figure $3 \mathrm{~A}$ ) and significantly reduced developmental viability $\left(F_{1,1157}=3905, P<0.00001\right.$; Supplementary Table S2L, Figure 3B). There was an interaction between strain and treatment for both measures of the developmental response to copper, indicating that although development time and developmental viability were negatively affected by copper exposure for the majority of strains, the magnitude of the effect of treatment varied among strains (development time: $F_{1,1157}=11.75, P<0.00001$; developmental viability: $F_{1,1157}=13.13, P<0.00001$; Supplementary Table S2, K-L, Figure 3, A and B).

Because we were primarily interested in the effects of copper on development time and developmental viability, we regressed out variation under control conditions from both developmental phenotypes (see Materials and Methods). Treatment-specific development time and treatment-specific developmental viability were correlated $\left(F_{1,98}=76.4, P<0.00001, R^{2}=44 \%\right.$; Supplementary Table S2M, Figure 3C), demonstrating that strains with longer development time on copper also had lower viability. Heritability was similar between treatment-specific development time $\left(H^{2}=87.7 \%\right)$ and treatment-specific developmental viability $\left(\mathrm{H}^{2}=87.7 \%\right)$.

Neither treatment-specific development time nor treatmentspecific developmental viability were correlated with adult copper resistance at an alpha level of 0.05 (treatment-specific development time: $F_{1,98}=0.16, P=0.69, R^{2}=0.02$; Supplementary Table $\mathrm{S} 2 \mathrm{~N}$, Figure 4A; treatment-specific developmental viability: $F_{1,98}=2.71, \quad P=0.10, \quad R^{2}=2.7 \%$, Supplementary Table S2O, Figure $4 \mathrm{~B}$ ). The lack of a significant correlation between either measure of the developmental response to copper and adult copper resistance could imply that copper resistance is influenced by life stage-specific mechanisms. However, because several other aspects of our adult and development assays differ (e.g., copper concentration and exposure time), we cannot rule out the possibility that the pattern we observe is also influenced by technical variation.

\section{QTL mapping of life stage-specific response to copper stress}

A principal goal of our study was to genetically dissect the response to copper stress. Using the DSPR, we identified a total of 12 QTL between the A and B panels that were associated with variation in adult copper resistance (Figure 5A, Table 1, and Supplementary Figure S6). Assuming that each QTL contributes to phenotypic variation in an additive manner, they collectively 
A

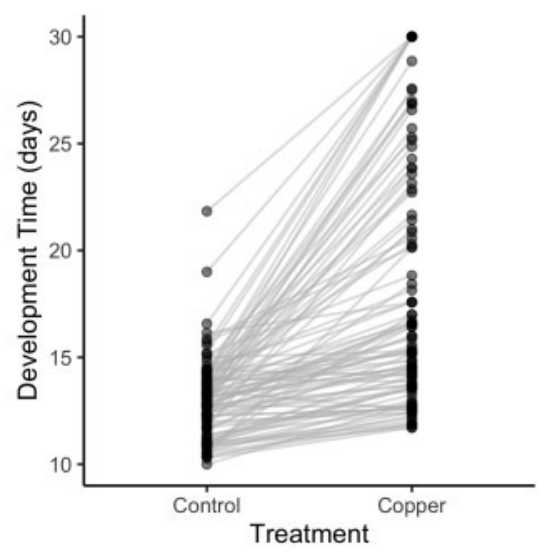

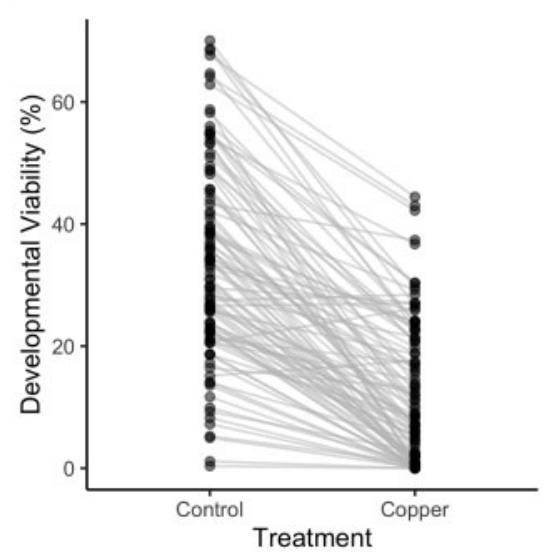

$\mathrm{C}$

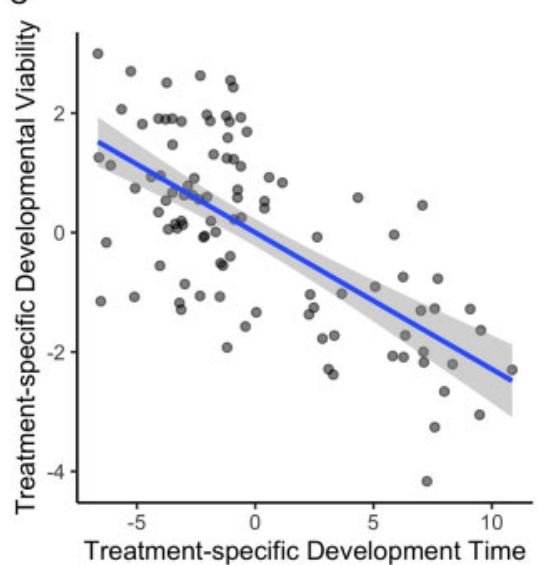

Figure 3 Copper exposure influences development time and viability in the DSPR. Development time (A) and developmental viability (B) were reduced in most strains by exposure to $2 \mathrm{mM} \mathrm{CuSO}_{4}$. (C) Copper treatment-specific developmental viability and development time (corrected for strain-specific variation in these responses on control food) were correlated $\left(P<0.00001, R^{2}=44 \%\right)$, indicating that strains with longer development time on coppersupplemented media also had lower viability. Points indicate the strain mean under each treatment condition. Gray shading indicates the $95 \%$ CI of the regression.
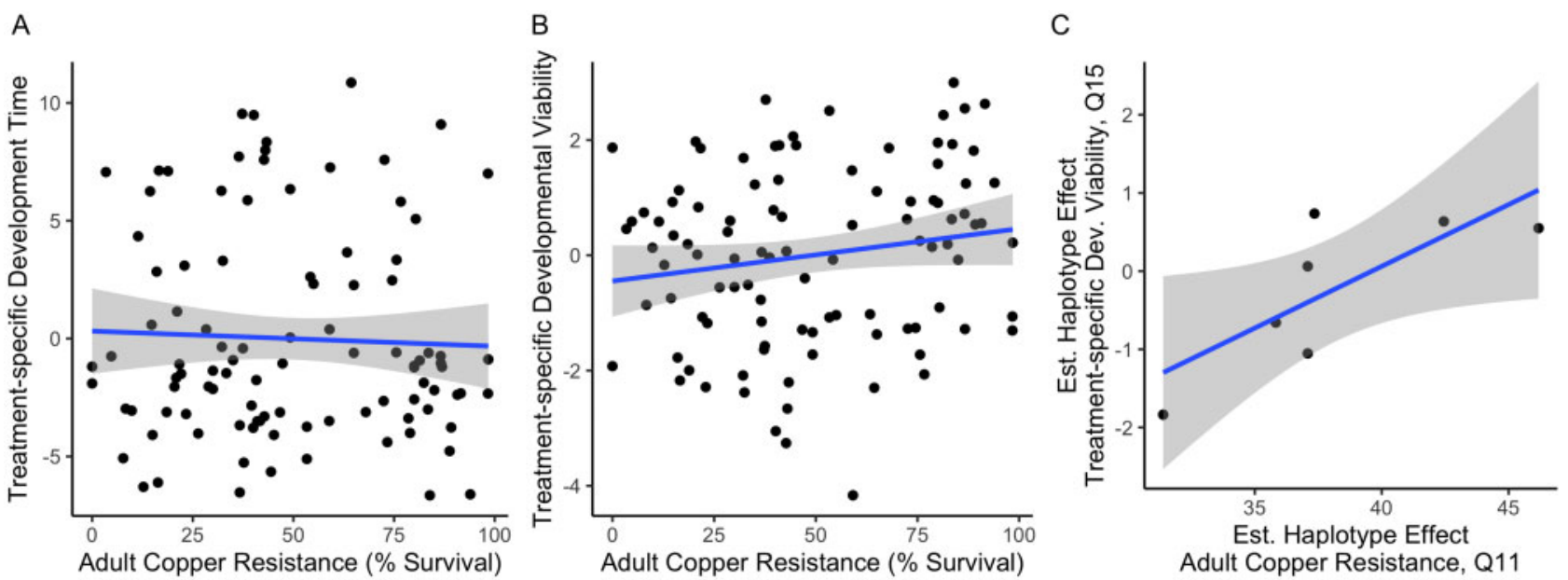

Figure 4 Copper resistance is not significantly correlated across life stages. (A) Copper treatment-specific development time was not correlated with adult copper resistance $(P=0.69)$. (B) Copper treatment-specific developmental viability and adult copper resistance were not correlated $(P=0.10)$. In both plots, points indicate strain means. Higher, positive values for treatment-specific development time and developmental viability indicate longer or higher development time or viability on copper, respectively. Gray shading indicates the $95 \% \mathrm{CI}$ of the regression between residual developmental response (corrected for variation in the response on control food) and adult female survival after $48 \mathrm{~h}$ on $50 \mathrm{mM} \mathrm{CuSO}_{4}$. (C) Founder haplotype effects for copper treatment-specific developmental viability and adult copper resistance estimated at a shared QTL position on chromosome 3R were significantly positively correlated $\left(P=0.04, R^{2}=59 \%\right)$. Gray shading indicates the $95 \% \mathrm{CI}$ of the regression between estimated founder haplotype effects at Q11 for adult copper resistance and at the equivalent genomic position for treatment-specific developmental viability, which resides within Q15.

explain a substantial amount of variation in adult copper resistance (A panel: 36.19\%; B panel: 27.93\%; Table 1). The genetic architecture of adult copper resistance was largely panel-specific, with only one QTL (Q3) overlapping between the mapping panels (Table 1, Figure 5A). Panel-specific genetic architecture of trait variation is consistent with several other studies that have mapped traits in both panels of the DSPR (Marriage et al. 2014; Najarro et al. 2017; Everman et al. 2019). This pattern is likely the result of using a different set of founders to establish each mapping panel (King and Long 2017) but may also reflect a lack of power (King et al. 2012a) or epistatic effects that influence our ability to detect all QTL underlying adult copper resistance in each panel. Several pairs of QTL are in close proximity (i.e., Q1/Q2, Q4/Q5, and Q8/ Q9-Figure 5A), with 0.7-0.53 MB separating the QTL intervals. However, the founder haplotype effects at these QTL pairs appear distinct, and there is no evidence for radical shifts in haplotype frequency between the QTL (Supplementary Figures S7-S9), so we consider them independent.

We did not find any QTL that contributed to food consumption, either on control or copper-supplemented food, or treatment-specific development time, even when using a relaxed $(\alpha=0.2)$ significance threshold. However, we did find three QTL that contributed to variation in treatment-specific developmental viability (Figure 5B). Given we only phenotyped 100 DSPR strains, power deficits certainly contribute to the low numbers of QTL identified for these traits (power to detect a QTL that explains $10 \%$ of phenotypic variation with 100 DSPR strains is $<20 \%$ ) (King et al. 2012a).

One treatment-specific developmental viability QTL (Q15) overlapped with a QTL (Q11) associated with adult copper resistance in the B panel (Figure 5B). The 2-LOD drop interval of Q11 fell entirely within the 3-LOD drop interval of Q15. To determine 
Table 1 Summary of QTL identified for response to copper stress by panel and life stage

Adult copper resistance QTL: A panel

\begin{tabular}{|c|c|c|c|c|c|c|}
\hline QTL & Peak LOD & Chr & Physical interval (Mb) ${ }^{a}$ & Genetic interval (cM) & Variance explained & No. genes ${ }^{b}$ \\
\hline Q1 & 10.13 & $2 \mathrm{~L}$ & $14.22-15.07$ & $49.52-60.62$ & 5.91 & 73 \\
\hline Q2 & 9.76 & $2 \mathrm{~L}$ & $15.77-16.38$ & $51.35-51.88$ & 5.70 & 68 \\
\hline Q3A & 11.3 & $2 \mathrm{~L}$ & 18.87-20.09 & $53.39-53.85$ & 6.56 & 154 \\
\hline Q6 & 8.01 & $2 \mathrm{R}$ & 7.03-7.72 & $63.56-64.89$ & 4.70 & 108 \\
\hline Q7 & 8.54 & $2 \mathrm{R}$ & $18.54-19.03$ & 100.24-102.17 & 5.01 & 92 \\
\hline Q10 & 6.88 & $3 R$ & $2.48-2.95$ & $47.56-47.74$ & 4.05 & 40 \\
\hline Q12 & 7.24 & $3 R$ & $21.01-21.46$ & $86.55-88.14$ & 4.26 & 78 \\
\hline
\end{tabular}

Adult copper resistance QTL: B panel

\begin{tabular}{|c|c|c|c|c|c|c|}
\hline QTL & Peak LOD & Chr & Physical interval $(\mathrm{Mb})^{\mathrm{a}}$ & Genetic interval (cM) & Variance explained & No. genes ${ }^{b}$ \\
\hline Q3B & 9.21 & $2 \mathrm{~L}$ & $17.95-20.31$ & $52.92-53.90$ & 5.24 & 259 \\
\hline Q4 & 9.44 & $2 \mathrm{R}$ & 1.41-3.69 & 54.99-57.07 & 5.36 & 247 \\
\hline Q5 & 8.46 & $2 \mathrm{R}$ & $4.08-6.58$ & $57.77-62.69$ & 4.82 & 353 \\
\hline Q8 & 7.88 & $3 \mathrm{~L}$ & $15.50-16.82$ & $42.90-44.06$ & 4.49 & 240 \\
\hline Q9 & 6.97 & $3 \mathrm{~L}$ & $17.35-19.47$ & $44.48-45.78$ & 3.99 & 224 \\
\hline Q11 & 7.05 & $3 R$ & $14.57-14.98$ & $63.92-65.00$ & 4.03 & 41 \\
\hline
\end{tabular}

Treatment-specific developmental viability QTL: B panel

\begin{tabular}{lcccccc}
\hline QTL & Peak LOD & Chr & Physical interval (Mb) & Genetic interval (cM) $^{\text {a }}$ & Variance explained $^{\text {c }}$ & No. genes \\
\hline Q13 & 7.11 & $2 \mathrm{R}$ & $13.30-13.95$ & $82.37-84.50$ & 27.9 \\
Q14 & 7.74 & $2 \mathrm{R}$ & $16.19-16.66$ & $90.86-92.45$ & 30.0 \\
Q15 & 8.65 & 3R & $14.52-15.06$ & $63.79-65.21$ & 60 & 32.8
\end{tabular}

a Physical intervals are based on FlyBase release 5 of the D. melanogaster reference genome.

b Protein-coding genes only. All genes including ncRNA and pseudogenes are included in Supplementary Table S4

c Estimates of QTL effects based on 100 DSPR strains are typically overestimated due to Beavis effects (Beavis et al. 1991; King and Long 2017), so estimates of the variance explained by the QTL mapped for developmental viability should be interpreted with care.

whether the QTL may represent the same locus, we compared the founder effects for both phenotypes at the peak position of the Q11 adult QTL (considering that the Q15 developmental QTL peak may not be well estimated given the sample size employed). We found that the estimated founder effects were significantly positively correlated $\left(F_{1,5}=7.11, \quad P=0.04, \quad R^{2}=59 \%\right.$; Supplementary Table S2P, Figure 4C), suggesting this position segregates for alleles that impact the response to copper stress in adults and developing individuals in a similar way. This result implies that the adult and developmental response to copper stress are not fully independent, as was suggested by the very weak phenotypic correlation between these traits (Figure 4, A and B). The level of overlap we observed between the adult and developmental viability responses may also be impacted by low sample size; however, founder haplotype frequencies in the full set of strains and the subset of 100 strains used to measure the developmental viability response are very similar across the genome (Supplementary Figure S10), suggesting that the subset of lines captures the same allelic diversity present in the full set.

\section{Genes implicated by mapped QTL}

Combined across panels, the QTL regions associated with adult copper resistance include a total of 1823 unique protein-coding genes. We identified potential candidate genes by searching among this list for genes with previous links to metal homeostasis, binding, metabolism, toxicity response, or transport, by executing QTL-focused, variant-by-variant association tests (Supplementary Figures S7-S9), and by examining variation in the estimated effects of founder haplotypes across QTL intervals (Supplementary Figures S7-S9). Of the 1823 genes, 10 genes have been previously linked to copper homeostasis, binding, chaperone activity, or development of specialized copper cells (Supplementary Table S4). Promising candidate genes include Syx5, Grx1, CG11825, Ccs, Sod3, and CG5235. Syntaxin 5 (Syx5), encompassed by Q2, Glutaredoxin 1 (Grx1), encompassed by Q9, and CG11825, encompassed by Q5, are all thought to play a role in copper ion homeostasis (Norgate et al. 2007, 2010; Mercer and Burke 2016). Syx5 is required for normal uptake of cellular copper, and it plays a critical role in copper ion homeostasis in D. melanogaster that is independent of other copper transporter proteins such as Ctr1A/B (Norgate et al. 2010). Similarly, Grx1 knockdown results in copper deficiency, and this gene may function as a mediator of copper transfer to chaperone proteins (Mercer and Burke 2016). CG11825 has been identified as a candidate for copper ion homeostasis in D. melanogaster by Norgate et al. (2007), but functional testing is lacking for this gene under copper stress conditions. The gene copper chaperone for superoxide dismutase (Ccs), found under Q5, is an important chaperone protein that shuttles copper ions to Sod1 under normal conditions (Culotta et al. 1997; Schmidt et al. 2000). Ccs was further supported as a promising candidate gene following variant-by-variant association tests of the QTL Q5 interval (Supplementary Figure S8). Genetic ablation of Ccs in D. melanogaster resulted in increased sensitivity to oxidative stress following paraquat exposure (Kirby et al. 2008); however, the effect of Ccs knockdown under copper stress conditions has not been assessed. Genes previously associated with copper ion binding include Sod3 (Q6) and CG5235 (Q8). While Sod3 functions as an extracellular receptor for copper ions and is protective against oxidative stress (Blackney et al. 2014), the link between CG5235 and copper is based only on prediction informed by GO (Gaudet et al. 2011).

In addition to these copper-associated genes, we identified 64 genes with functions related to homeostasis or detoxification of zinc, two genes involved with manganese regulation, and 19 genes involved in binding unspecified metals. Of particular interest among these genes are Catsup (Q3), ZnT41F (Q4), and stl (Q7), which are all linked to zinc transport or detoxification (Yepiskoposyan et al. 2006; Ozdowski et al. 2009; Lye et al. 2013; 
A

Adult Cu Resistance
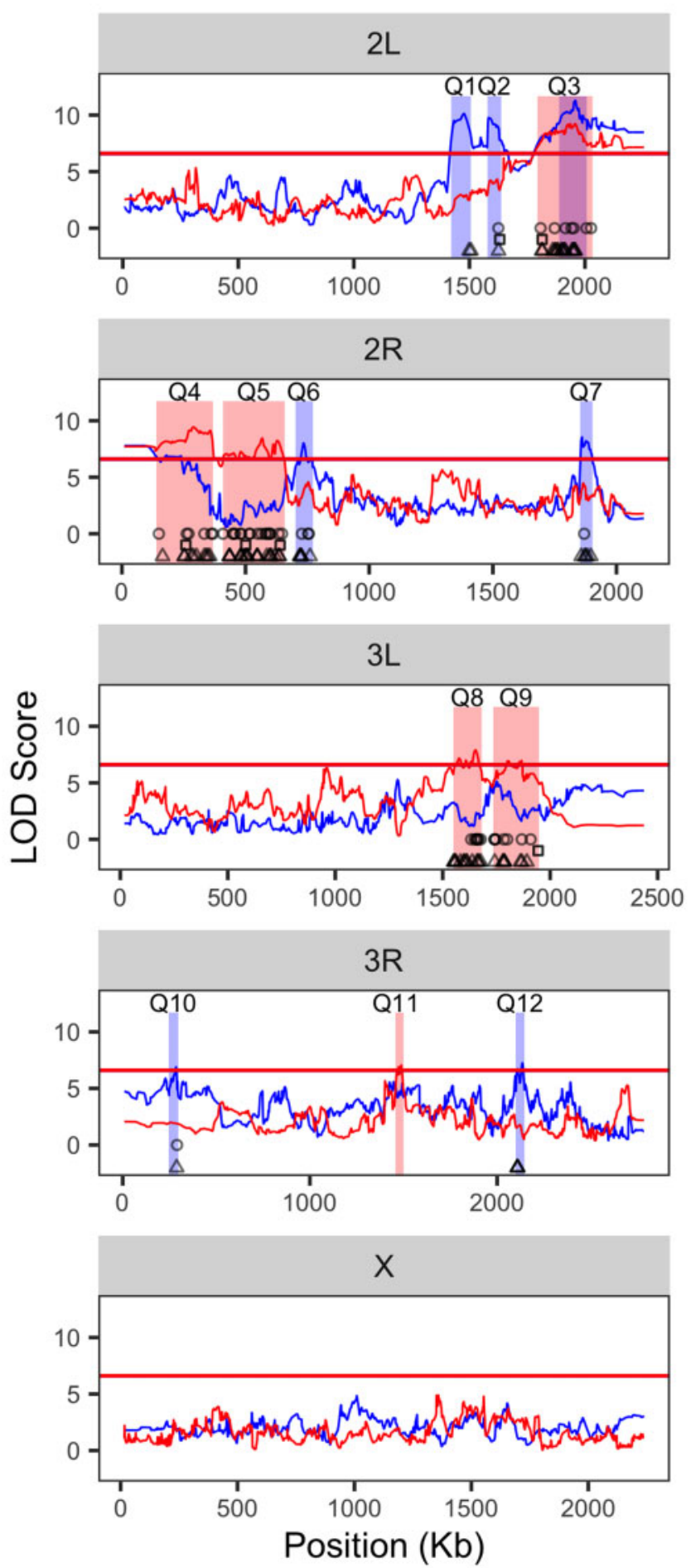

B Treatment-specific Dev. Viability
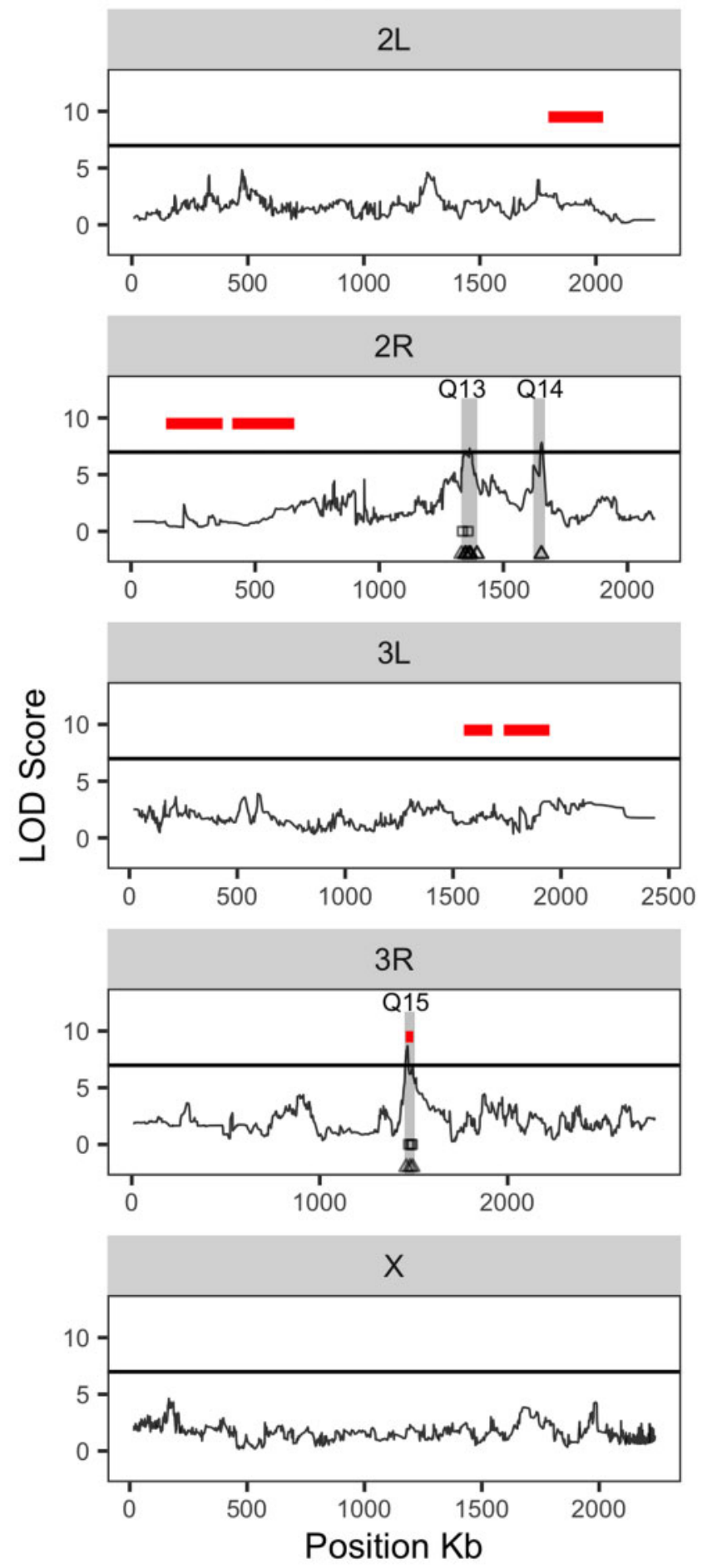

Figure 5 QTL associated with adult copper resistance and treatment-specific developmental viability. QTL associated with variation in adult copper resistance (A) and copper treatment-specific developmental viability (B). (A) We detected several QTL in the A (blue) and B (red) DSPR panels. Most QTL were panel specific with one QTL (Q3) overlapping between panels. Red and blue bars represent the 2-LOD drop intervals for each QTL. (B) QTL mapped for copper treatment-specific developmental viability. One QTL (Q15) for developmental viability overlapped with Q11, associated with adult copper resistance in the (B) panel. Red horizontal lines represent the 2-LOD drop intervals for the six QTL associated with the (B) panel adult survival response to copper, and gray bars represent the 3-LOD drop for the three QTL associated with treatment-specific developmental viability (Table 1). The horizontal lines in each plot represent permutation-derived 5\% critical thresholds (the thresholds for each panel in (A). are nearly identical, leading to the lines overlapping.) Round points indicate DE genes influenced by resistance class, triangle points indicate DE genes influenced by treatment, and square points indicated DE genes that are shared between the treatment and resistance class models and that fall within the QTL boundaries. DE genes across the rest of the genome are not shown. 
Navarro and Schneuwly 2017), trpl (Q5) and DCP2 (Q8), which are hypothesized to be involved in manganese ion binding (Thurmond et al. 2019), and swm (Q3), babo (Q5), and whd (Q5), which are thought to be involved in binding of unspecified metal ions based on GO prediction (Gaudet et al. 2011; Thurmond et al. 2019). Similar to Ccs, the gene trpl was supported by variant-byvariant association tests of the Q5 interval (Supplementary Figure S8), further suggesting this gene is a promising candidate for functional testing under copper conditions.

The three QTL associated with copper treatment-specific developmental viability spanned a total of 247 unique proteincoding genes. Of these genes, none had functions previously linked to copper. However, eight genes were associated with zinc ion binding, and two were linked to metal ion binding through GO prediction (Supplementary Table S4; Gaudet et al. 2011; Thurmond et al. 2019). Most notable among the genes identified by treatment-specific developmental viability QTL was mekk1 (Q15), which was demonstrated though gene knockdown to be the primary activator of JNK signaling under cadmium stress in Drosophila S2 cells (Ryabinina et al. 2006). Although the Q15 developmental viability QTL overlaps with the adult copper resistance QTL Q11, mekk1 is only present within the interval implicated by Q15. Given that mekk1 is within $11.2 \mathrm{~kb}$ of the Q11 2-LOD drop interval, this gene may still be a plausible candidate for adult copper resistance.

\section{Differential gene expression due to treatment and resistance class}

Allelic effects on variation in complex traits are commonly mediated by regulatory variation (Roelofs et al. 2006; Ruden et al. 2009; Boyle et al. 2017; GTEx Consortium et al. 2018). We used an RNA sequencing approach to examine the effects of copper stress on gene regulation and to assess any differences in this response between genotypes with high or low adult copper resistance. We sequenced mRNA from whole females from six high (79-98\%) and four low (0-18\%) adult copper resistance strains from the B panel following a 9-h exposure to control (water) and $50 \mathrm{mM} \mathrm{CuSO}_{4}$ conditions. A primary goal was to determine whether there are consistent differences in gene expression between high and low resistance classes of strains when exposed to copper stress, so we treated each strain as a replicate of the high and low resistance classes. We performed differential expression analysis by first testing whether an interaction between treatment and resistance class influenced gene expression (interaction model: $\sim \mathrm{TRT} \times \mathrm{RES}$ us reduced model: $\sim \mathrm{TRT}+\mathrm{RES})$. We followed this initial analysis with three subsequent analyses to test the additive effects of treatment and resistance class on gene expression: (1) Full model: $\sim$ TRT + RES us reduced model: $\sim 1)$, (2) Treatment Model: $\sim$ TRT + RES us reduced model: $\sim$ RES), and (3) Resistance Class Model: $\sim$ TRT + RES us reduced model: $\sim$ TRT. The treatment and resistance class models allowed us to identify specific genes that were influenced primarily by either of these two main effects.

The interaction between treatment (control us $50 \mathrm{mM} \mathrm{CuSO}_{4}$ ) and resistance class was not significant at a $5 \%$ FDR or at a relaxed FDR of $20 \%$. Lack of a significant interaction is likely due to limited power as a result of small sample size. After additional filtering (see Materials and Methods), we identified 1589 genes that were DE across treatment and adult copper resistance class with the full model (full model: TRT + RES us reduced model: $\sim 1$ ). We used PCA with quantile-normalized filtered TPM data from these 1589 genes and found a pronounced effect of treatment on gene expression, with a more subtle effect of resistance class (Figure 6, Supplementary Table S5). The relatively subtle effect of

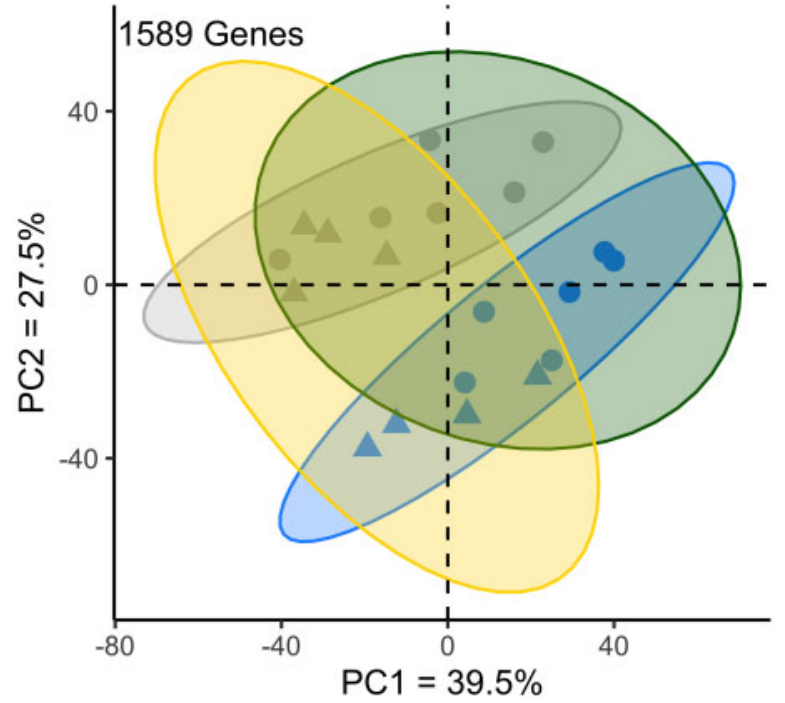

Figure 6 Differentially expressed genes cluster by treatment and resistance class. Principal components analysis of significantly differentially expressed genes (quantile-normalized filtered TPM) identified by the full model (full model: TRT + RES us reduced model: 1). The effect of treatment was pronounced among samples, while the effect of resistance level was more subtle. Ellipses indicate the equivalent of a 95\% confidence interval. Blue indicates copper-exposed samples, gray indicates control-exposed samples, green indicates resistant strains, yellow indicates sensitive strains. Triangle points indicate sensitive strains; circles indicate resistant strains.

resistance class on gene expression likely also contributes to the lack of a significant treatment by resistance class interaction.

To further explore how each of the main effects of the differential expression model influence gene expression, and to identify sets of genes that were influenced primarily by treatment or resistance class, we tested the effects of treatment and resistance class separately. Treatment alone (treatment model: $\sim$ TRT + RES us reduced model: $\sim$ RES) primarily contributed to differential expression in 848 genes, and adult copper resistance alone (resistance model: $\sim$ TRT + RES us reduced model: $\sim$ TRT) primarily contributed to differential expression in 466 genes. The vast majority of genes influenced by treatment and resistance class were included among the 1589 genes identified with the full model (92\% of genes identified with the treatment model, $91 \%$ of genes identified with the resistance model). Of the 848 and 466 genes identified with the treatment and resistance class models, 58 genes were shared. The proportion of shared genes increased when a more relaxed significance threshold (20\% FDR) was used in the treatment and resistance class models, and the estimated effects of resistance class and treatment on gene expression in each DE gene list were weakly positively correlated (Treatment Model: $R^{2}=8 \%$; Resistance Class Model: $R^{2}=2 \%$ ). While the $\mathrm{DE}$ gene lists attributed to treatment and resistance class are not fully independent, they represent sets of genes for which the primary source of variation is either treatment or resistance class.

To broadly characterize DE genes identified in the treatment and resistance class models, we performed GO analysis with FlyMine (Lyne et al. 2007) for each gene list separately. GO analysis of each complete DE gene list is summarized in Supplementary Table S6. Briefly, 111 GO terms were identified from the full model DE gene list and included terms related to cell organization, cell cycle, and metabolism among the top 10 (Supplementary Table S6). Enrichment for 23 GO terms including those related to cytoplasmic translation, ribosome biogenesis, 
and RNA processing was observed for the 848 genes influenced by treatment (Supplementary Table S6). Fifty-nine GO terms were identified from the $466 \mathrm{DE}$ genes influenced by resistance class. Top among these GO terms were those related to ATP synthesis, cellular respiration, and mitochondrial function (Supplementary Table S6). GO analysis of this set of 58 genes revealed enrichment for female gamete generation [G0:0007292] $(P=0.006)$, and no genes had any apparent connection to copper or metal ion homeostasis. Notably, no enrichment was observed for any GO term related to metal ion homeostasis or detoxification when the total DE gene lists were considered (but see below).

Many of the DE genes influenced by treatment and/or resistance class fell within the QTL intervals for adult copper resistance or treatment-specific developmental viability (Figure 5). Of the 848 genes with DE due to treatment, 87 (11\%) overlapped with QTL intervals, and of the 466 genes with DE due to resistance class, 62 genes (13.3\%) overlapped with QTL intervals. Of the 58 genes shared between the treatment and resistance class models, 12 genes (20.6\%) overlapped with QTL intervals (Figure 5). DE genes from the treatment and resistance class models were not more likely than expected by chance to fall within QTL intervals $\left(\chi^{2}=14.5, \mathrm{df}=14, \mathrm{P}=0.41\right)$, although this does not preclude the possibility that those DE genes within mapped QTL are strong candidates that may contribute to copper resistance.

To further explore the influence of resistance class on gene expression, we calculated the average change in gene expression following copper exposure for each of the $1589 \mathrm{DE}$ genes from the full model using the same filtered TPM data used for PCA above. The absolute values of these data were then log transformed to reduce spread, and the sign of the change in gene expression was restored by multiplying the result by 1 or -1 .

Of the $848 \mathrm{DE}$ genes identified in the treatment model, there was a roughly even split between copper-induced and copperrepressed genes, with no difference between the resistance classes [Kolmogorov-Smirnov (KS) test: $D=0.05, P=0.33$; Figure 7A]. Among the top 20 most highly induced genes under copper conditions in both resistant and sensitive classes were several MTs
(MtnA, MtnC, MtnD, MtnE) as well as two genes that comprise a major iron storage complex (Fer1HCH and Fer2LCH). Because these genes and other genes with DE due to treatment were induced in sensitive and resistant strains to similar degree, we suggest that variation in sensitivity to copper is not due to a failure to induce expression of genes with protective functions against copper ions. Among the $466 \mathrm{DE}$ genes identified in the resistance model, gene induction by copper was more frequently observed in sensitive strains compared to resistant strains (KS test: $\mathrm{D}=0.27, \mathrm{P}<0.00001$; Figure $7 \mathrm{~B})$. The top 20 most highly induced genes under copper conditions in sensitive strains included several genes that are involved in mitochondrial structure, function, and energy synthesis (e.g., Ald1, levy, sesB, Mpcp1, COX5A, ATPsynb), suggesting more sensitive strains may be characterized by a greater susceptibility to oxidative stress.

\section{Cluster analysis of DE genes}

The patterns of copper-influenced gene expression across treatment and resistance class observed in Figure 7 raise the question of whether there are co-regulated sets of genes that distinguish resistant and sensitive strains under copper and control conditions. For example, the presence of metal-associated genes among the those induced by treatment may signal a larger network of genes that are co-regulated in response to heavy metal stress. To identify any such co-regulated groups, we used Clust (Abu-Jamous and Kelly 2018) to identify non-overlapping clusters of genes from the 848 genes influenced by treatment and the 466 genes influenced by resistance class using quantile-normalized, filtered TPM data.

Clust identified three clusters of co-regulated genes with $\mathrm{DE}$ due to treatment (Figure 8A, Supplementary Figure S11A). Treatment clusters 1 (101 genes) and 2 (17 genes) consisted primarily of genes that were induced by copper exposure, while treatment cluster 3 (17 genes) consisted primarily of genes that were copper-repressed (Figure 8A, Supplementary Figure S11A). The top GO terms for treatment clusters 1 and 3 revealed enrichment for genes involved in processes unrelated to metal ion
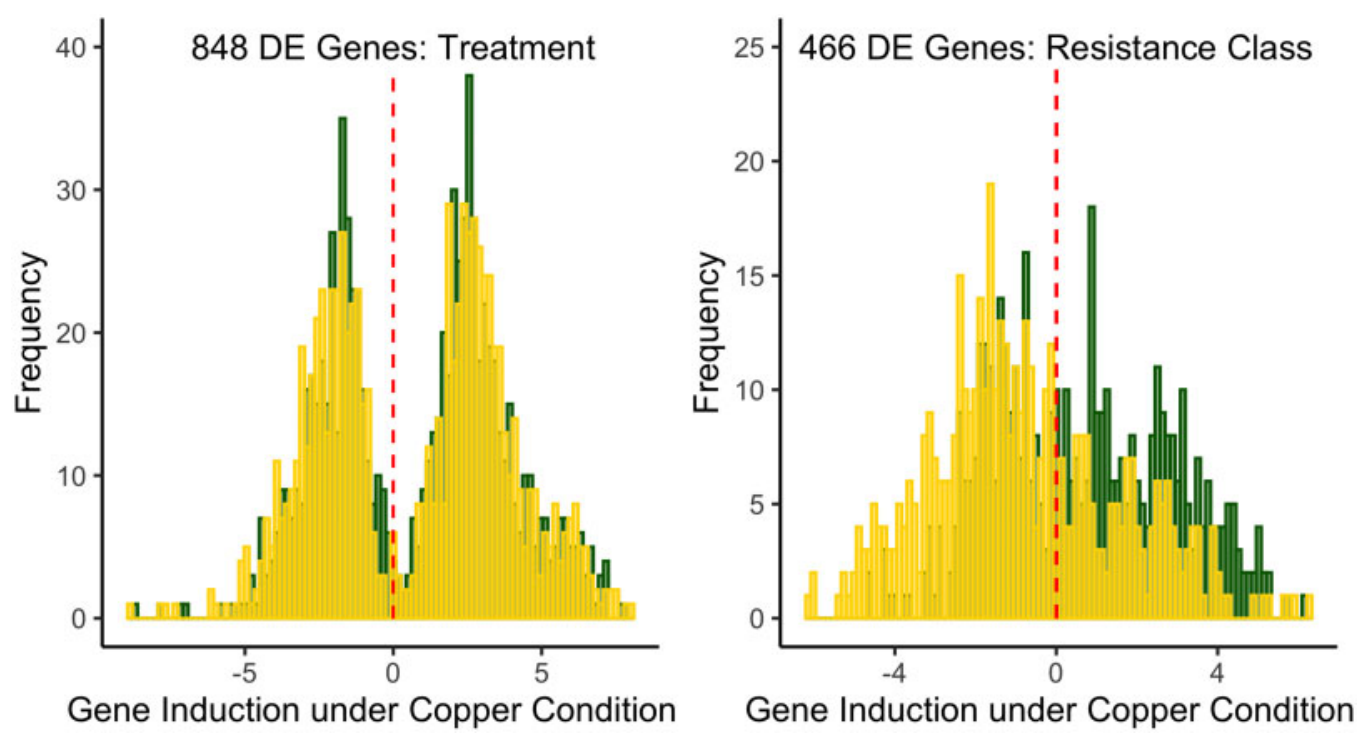

Figure 7 Copper-induced changes in gene expression vary by treatment and resistance class. Induction of genes under copper conditions with DE identified from the treatment model (A) and the resistance model (B). (A) The effect of treatment on DE highlights that roughly equal numbers of genes were induced or repressed under copper conditions (KS test: $D=0.05, P=0.33$ ). (B) Among DE genes identified by the resistance model, genes were more likely to be induced by copper exposure in sensitive strains compared to resistant strains (KS test: $D=0.27, P<0.001$ ). In each plot, yellow bars indicate gene expression in sensitive strains and green bars indicate gene expression in resistant strains. 

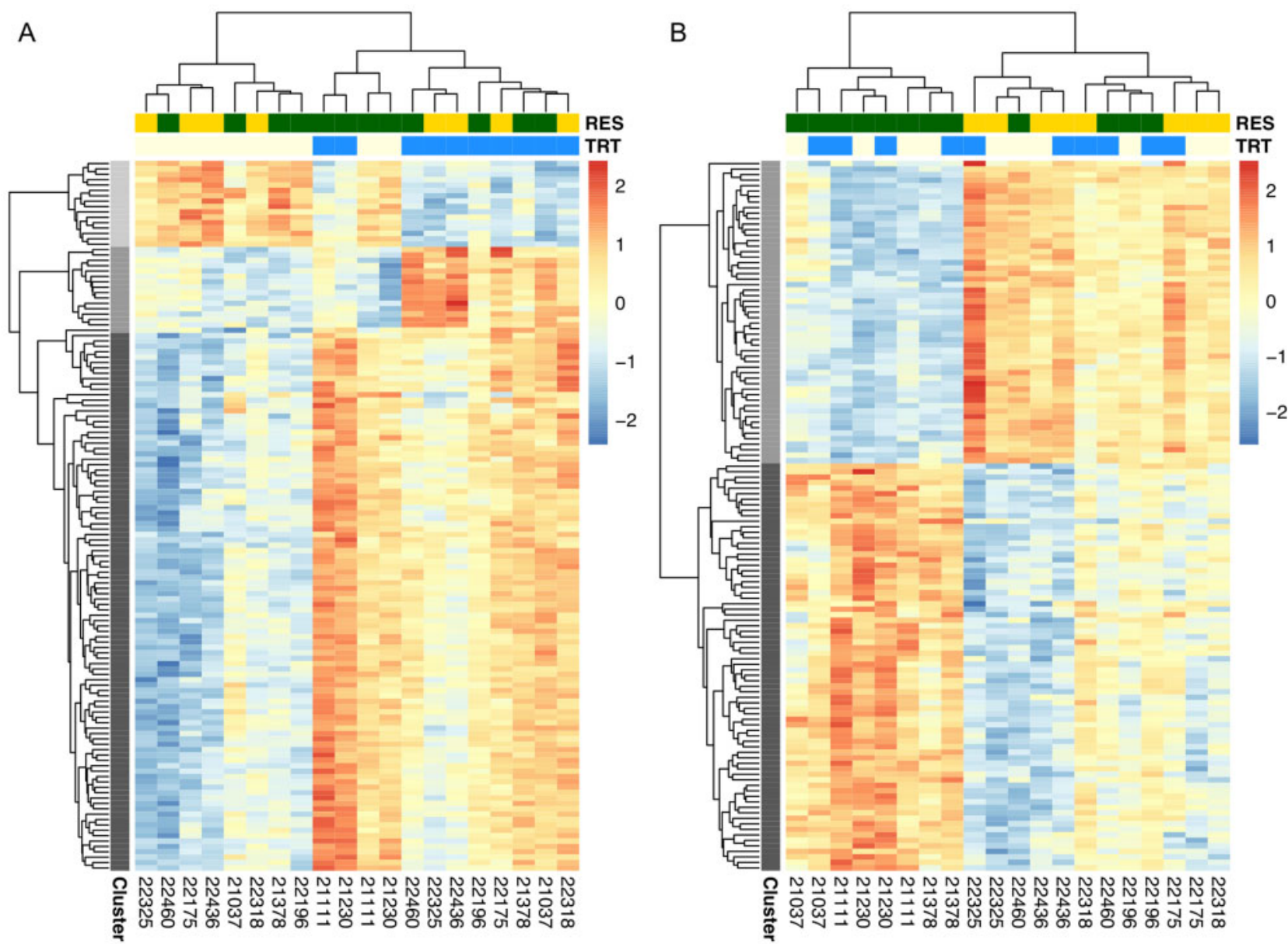

Figure 8 Heatmap of differentially expressed genes due to treatment and resistance class. Three clusters were identified among the genes with DE due to treatment (A), and two clusters were identified among genes with DE due to resistance class (B). Expression is presented following z-normalization of the 132 and 129 genes that clustered from the treatment gene set and the resistance class gene set, respectively. Cluster is mapped to the $y$-axis dendrogram [Cluster $1(A, B)=$ dark gray, Cluster $2(A, B)=$ medium gray, Cluster $3(A)=$ light gray], and resistance class (green = resistant, yellow $=$ sensitive) and treatment (blue $=$ copper-exposed, cream $=$ control) are mapped to the $\mathrm{x}$-axis dendrogram.

homeostasis or response (e.g., cell cycle, RNA processing; Supplementary Table S6). However, treatment cluster 2 was enriched for genes involved in iron import, transport, and detoxification of iron and inorganic compounds (Supplementary Table S6). Among these genes were Fer $1 \mathrm{HCH}$ and Fer2LCH, which are primarily involved with iron storage, but may also interact with copper ions during protein assembly (Huard et al. 2013). Despite these GO terms suggesting treatment cluster 2 is enriched for genes that respond to toxic metal ion exposure, only one of the genes (Gclc) has been previously directly linked to copper (Gclc interacts with the copper transport proteins Ctr1A and ATP7; Mercer et al. 2016). Treatment clusters 1 and 3 included 17 and four genes, respectively, that were implicated by adult copper resistance-associated QTL. The gene dnk in treatment cluster 1 was also implicated by the treatment-specific developmental viability QTL Q15. Two of the genes (CG11878 and CG5506) identified in treatment cluster 2 were implicated by adult copper resistance-associated QTL intervals. One gene, CG5506, was empirically demonstrated to interact with Fer2HCH (Guruharsha et al. 2011); however, neither gene has been previously associated with copper exposure.

Clust identified two clusters of co-regulated genes that were DE due to resistance class (Figure 8B, Supplementary Figure S11B). Resistance cluster 1 (75 genes) was primarily enriched for genes involved in cell cycle processes (Supplementary Table S6), and 11 genes were also implicated by adult copper resistance QTL. Resistance cluster 2 (56 genes) included genes that were more often copper-induced in sensitive strains and copperrepressed in resistant strains (Figure 8B, Supplementary Figure $\mathrm{S} 11 \mathrm{~B})$. Resistance cluster 2 was enriched for two broader categories of GO terms including several related to muscle structure (e.g., myofibril assembly [GO:0030239], P<0.00001) and mitochondrial function and energy synthesis (e.g., ATP metabolic process [GO:0046034], P < 0.00001). Resistance cluster 2 also included genes involved in inorganic ion homeostasis (GO:0098771), although enrichment for this GO term was weak $(P=0.05)$. Of the genes involved in inorganic ion homeostasis, three (CG14757, trpl, and sesB) are particularly noteworthy given that all three genes are copper-induced in sensitive strains and copper-repressed in resistant strains, and two have been previously linked to metal ion homeostasis (Figure 9). Exposure of the Drosophila S2 cell line to $2 \mathrm{mM} \mathrm{CuSO}_{4}$ resulted in increased expression of CG14757, indicating that this gene is responsive to copper stress (Norgate et al. 2007); however, its exact function relative to the toxic effects of copper has not been elucidated. The gene trpl is predicted to be involved in manganese ion binding (Thurmond et al. 2019) and was included in the adult copper resistance-associated QTL Q5 we mapped. sesB is a mitochondrial transporter gene that was 


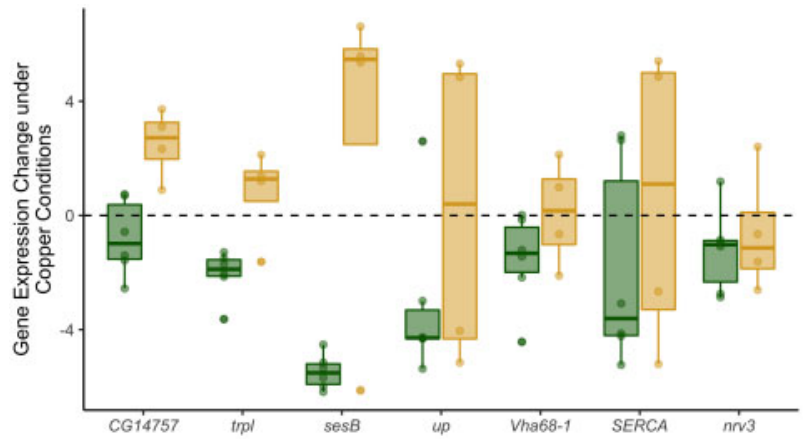

Figure 9 Copper-affected expression of genes involved in inorganic ion homeostasis that were included in resistance cluster 2. Resistant strains are shown in green, sensitive strains are shown in yellow.

demonstrated to be important for protection against oxidative stress through gene knockdown in D. melanogaster (Terhzaz et al. 2010). Other genes included in this group (up, SERCA, and nru3; Figure 9) are involved in transport of calcium, sodium, and potassium (Domingo et al. 1998; Gaudet et al. 2011) or are thought to be involved in ATP metabolism (Vha68-1) (Thurmond et al. 2019). In addition to trpl, two other genes from resistance cluster 2 were implicated by adult copper resistance QTL.

\section{RNAi knockdown of candidate genes associated with adult copper survival}

Several genes with links to copper or metal ion homeostasis were implicated by QTL or were DE due to treatment or resistance class (see above) (Supplementary Table S3). We chose 16 genes to functionally test using RNAi knockdown. QTL-implicated genes included Catsup and swm (Q3), ZnT41F (Q4), CG11825, whd, babo, and CCs (Q5), stl (Q7), DCP2 and CG5235 (Q8). We also tested trpl (Q5), which, along with Mul, was among the DE genes influenced by resistance class. Because Ccs and both Sod1 and Sod2 closely interact, we tested Sod1/2 even though these genes were not implicated by either QTL or RNAseq. From genes with DE due to treatment, we tested MtnC and CG10505. Of this set of genes, only Sod1, MtnC, and Mul have been previously specifically linked to copper stress (Calap-Quintana et al. 2017). Ccs, CG5235, Sod1, CG11825, and CG10505 are all associated with copper transport or binding. The remaining candidate genes (trpl, DCP2, whd, stl, swm, babo, Catsup, and ZnT41F) have not been experimentally linked to copper stress but are associated with metal ion binding or homeostasis.

Genes were tested using TRiP UAS RNAi strains (Perkins et al. 2015) that were crossed to a background with a ubiquitously expressed Gal4-expressing driver resulting in knockdown in the whole animal throughout all developmental stages, and to a background with an adult, anterior midgut-specific Gal4-expressing driver (Buchon et al. 2013). Copper absorption occurs in the copper-accumulating region of the middle midgut (Filshie et al. 1971; Calap-Quintana et al. 2017); however, the majority of candidate genes we tested (trpl, Ccs, CG10505, Mul, swm, babo, Catsup, CG11825, Sod1/2, and ZnT41F) are expressed throughout the midgut, including the anterior midgut region (Buchon et al. 2013).

In general, more genes influenced copper resistance when they were knocked down in the whole animal compared to solely in the anterior midgut (Figure 10). Of the candidate genes with known associations with copper, Ccs, CG5235 (b), MtnC (b), and Sod1 reduced copper resistance relative to the control when knocked down in the whole animal using the ubiquitous driver (Figure 10A). Inconsistent effects of ubiquitous CG5235
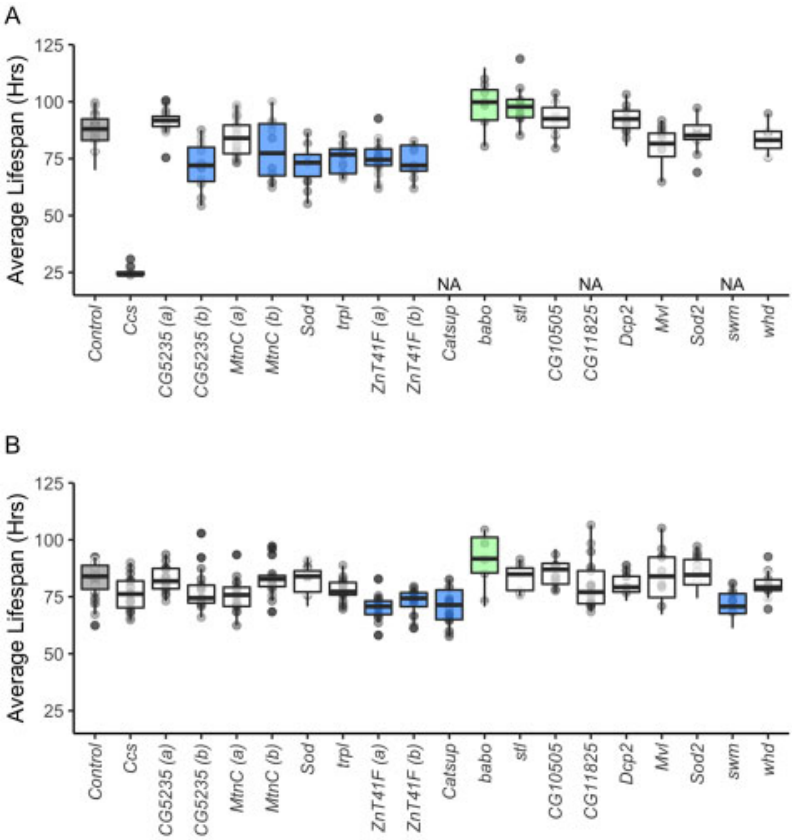

Figure 10 RNAi knockdown of candidate genes. Average lifespan of TRiP UAS RNAi knockdown strains crossed to a ubiquitous Gal4-expressing driver (A) and to an anterior-specific Gal4-expressing driver (B). (A) Increased susceptibility was observed with knockdown of CCs, CG5235 (b), MtnC (b), Sod, trpl, and ZnT41F with the ubiquitous Gal4 driver. Knockdown of babo resulted in increased resistance to copper toxicity relative to the control. (B) Knockdown in the anterior midgut of Catsup, swm, and ZnT41F resulted in increased susceptibility to copper toxicity, while knock down of babo increased resistance relative to the control. In each plot, gray shading indicates the control, green shading indicates increased resistance to copper, blue shading indicates decreased resistance, and no shading indicates lack of a significant difference based on an experiment-wide $a=0.05$. Three candidate gene TRiP strains (swm, Catsup, and CG11825) produced too few flies to test when crossed to the ubiquitous Gal4-expressing driver and were thus excluded from our analysis. We tested multiple TRiP UAS RNAi strains for genes CG5235 [CG5235 (a), CG5235 (b)], MtnC [MtnC (a), MtnC (b)], and ZnT41F [ZnT41F (a), ZnT41F (b)] to assess the consistency in the effect of gene knockdown on copper survival (Supplementary Table S3).

knockdown may be influenced by vector efficiency; the knockdown vector for CG5235 (a) is a long dsRNA vector (VALIUM10), while the knockdown vector for CG5235 (b) is a shRNA vector (VALIUM20). Both TRiP strains for MtnC used the same vector (VALIUM20) (Perkins et al. 2015); however, these two strains target $\mathrm{MtnC}$ at different locations within the gene, and knockdown efficiency may differ between the two sites. Off-target effects and leaky gene expression are additional potential sources of error in RNAi knockdown experiments. However, TRiP RNAi strain attributes have been shown to improve efficiency with limited offtarget effects (Ni et al. 2009, 2011; Perkins et al. 2015). The majority of TRiP strains used in our study implement short hairpin RNAs with the VALIUM20 vector, which has been demonstrated to have strong knockdown effects (Ni et al. 2011). Knockdown of copperassociated genes in the anterior midgut did not influence copper resistance relative to the control, suggesting that reduced expression of CCS, CG5235, Sod1, CG11825, and CG10505 in this limited region of the midgut does not hinder the fly's ability to cope with copper stress (Figure 10B).

The candidate gene ZnT41F consistently reduced copper resistance relative to control when knocked down in the whole animal and in the anterior midgut. While ZnT41F was previously shown to indirectly affect zinc homeostasis (Yin et al. 2017), the role it 
plays in copper ion homeostasis has not been described. Similarly, Catsup and swm, which have not been previously linked to copper, reduced copper resistance when knocked down in the anterior midgut. That knockdown of these genes in the whole animal did not influence copper resistance suggests these genes interact with copper soon after ingestion, although this would require additional follow-up to confirm. Interestingly, knockdown of babo in both the whole animal and the anterior midgut increased copper resistance relative to the controls (Figure 10). Knockdown of stl in the whole animal had a similar effect. Both genes are predicted to be involved in metal ion binding (Gaudet et al. 2011; Thurmond et al. 2019), but any additional evidence linking them to the detoxification of heavy metal ions under stressful conditions is lacking.

\section{Discussion \\ Variation in heavy metal stress is influenced by a complex genetic architecture}

Drosophila melanogaster has been an important model for elucidating the roles of genes involved in the response to copper and other heavy metals (e.g., Egli et al. 2003, 2006a, 2006b; Balamurugan et al. 2004, 2007; Yepiskoposyan et al. 2006; CalapQuintana et al. 2017). In our study, we used this model to investigate the role of allelic and expression variation in resistance to the heavy metal copper. We used a combination of QTL mapping and RNA sequencing to characterize allelic and gene expression variation that influences resistance to copper stress in strains from the multiparental DSPR mapping panel. In comparison with previous reports investigating the genetic architecture of copper and resistance to other heavy metals in plants (Macnair 1993; Selby and Willis 2018), the genetic architecture of copper resistance in D. melanogaster appears to be more complex. Where one to three QTL were identified for heavy metal resistance in several plant species including Mimulus guttatus, wheat, and corn (Allen 1971; Macnair 1983, 1993; MacNair et al. 1993; Bálint et al. 2007; Selby and Willis 2018), we identified 12 QTL that underlie variation in adult copper resistance (Figure 5A), and found that the diverse DSPR strains varied widely in survival following exposure to copper stress (Figure 1).

Part of the difference in apparent complexity underlying the response to copper is likely due to the higher power of our mapping panel, which employs a much larger number of genetically diverse strains coupled with higher genetic marker density compared to the mapping populations used in many plant studies (e.g., Courbot et al. 2007; Willems et al. 2007; King et al. 2012a). Secondly, the structure of the DSPR may be particularly conducive for detecting allelic variation in genes that influence the response to copper stress given the global sampling of founder strains used to generate the DSPR (King et al. 2012a), which may capture more of the natural variation for copper resistance than that present in any one natural population. Third, in contrast to natural populations which are often of interest because of their proximity to heavy metal pollution (e.g., Allen 1971; Macnair 1983; Ramirez et al. 2005; Turner et al. 2010; Wuana and Okieimen 2011; Wright et al. 2015; Arnold et al. 2016), the DSPR is likely naïve to any form of heavy metal selection or stress. Strong selection for heavy metal resistance could reduce variation at causative genes and lead to an apparent reduction in the complexity of resistance (Arnold et al. 2016).

The level of genetic complexity for copper resistance described in our study is consistent with reports of metal resistance in flies, yeast, and worms where measures of resistance were conducted in other heavy metal-naïve mapping populations. The DGRP (Mackay et al. 2012), another large D. melanogaster mapping panel, was used to demonstrate a complex genetic architecture for heavy metal exposure through GWA (genome-wide association) and extreme QTL mapping (i.e., sequencing and comparing pools of individuals with divergent phenotypes) in adult and developing life stages (Montgomery et al. 2014; Zhou et al. 2016, 2017). In these studies, tens to hundreds of genes have been implicated in natural genetic screens for lead (Zhou et al. 2016, 2017), cadmium (Zhou et al. 2017), and methylmercury (Montgomery et al. 2014). In Saccharomyces cerevisiae using several extreme QTL mapping pools, Ehrenreich et al. (2010) demonstrated that more than 20 distinct loci were associated with resistance to cadmium and nearly 40 loci were associated with copper resistance. In Caenorhabditis elegans, Evans et al. (2018) found 4, 6, and 6 QTL associated with the response to cadmium, copper, and silver, respectively.

In addition to a complex genetic architecture underlying the response to any one metal stressor, it is also possible that genes that are linked to one metal may play a role in the response to other metals through pleiotropic effects or as members of gene networks. Previous studies in yeast and worms have found limited evidence of pleiotropy underlying the response to multiple chemical stressors (Ehrenreich et al. 2010; Evans et al. 2018). However, we noted that several promising candidate genes associated with copper in our study had been previously linked to or were predicted to interact with metals other than copper [e.g., swm (Q3), babo (Q5), and stl (Q5); Figure 10, Supplementary Table S3]. Although we did not measure resistance to multiple heavy metal stressors in our study, RNAi knockdown of genes linked to other metals did impact copper resistance (Figure 10). Pleiotropic gene effects inferred from our RNAi analyses may be the result of metal-sensitive genes responding to a generalized set of cytotoxic effects stemming from production of ROS caused by heavy metal toxicity (Uriu-Adams and Keen 2005). This hypothesis is further supported by evidence of copper-induced expression of genes involved in oxidative stress response in sensitive strains that are repressed in resistant strains (e.g., sesB, Figure 9). However, additional tests of the response of DSPR strains to a diverse set of heavy metals is needed to fully understand whether the noncopper candidate genes we identified have correlated effects on resistance to other heavy metals.

Additional functional testing of the candidate genes highlighted in our study would also help illuminate the role of natural variation in the candidate genes we identified in copper resistance in wild populations. Our study presents evidence that RNAi knockdown of a set of candidates alters copper resistance; however, this approach does not allow us to investigate the effects of naturally occurring allelic variation, and only indirectly points to the likelihood these genes harbor segregating variation impacting response to copper. Reciprocal hemizygosity experiments (Stern 2014) would allow us to test putatively functionally distinct alleles present among the DSPR founder strains (Supplementary Figure S6), and genome editing tools such as CRISPR-Cas9 can be used to generate null mutations of candidate genes in high and low resistance DSPR strains.

\section{Consistency in the genetic architecture of copper resistance across life stages}

Genes that are involved in copper homeostasis in D. melanogaster adults have in some cases also been shown to regulate copper in larvae. For example, exposure of larvae to $\mathrm{CuSO}_{4}$ induces expression of MTs (Egli et al. 2003, 2006b), and we demonstrated that 
copper induces higher MT expression in adults (Supplementary Table S5). Knockdown of copper transporter genes in the CTR family alters copper homeostasis in both larvae and adult flies as well (Zhou et al. 2003; Turski and Thiele 2007). Our goal was to understand the relationship between adult copper resistance and the effect of copper stress on development in a set of genetically diverse $D$. melanogaster strains.

Similar to previous reports, we found that copper stress delayed development and reduced viability (Zhou et al. 2003; Bahadorani and Hilliker 2009; Pölkki 2016) although to differing degrees among the DSPR strains (Figure 3), suggesting that as with adult copper resistance, treatment-specific development time and developmental viability are genetically variable. Despite the lack of a statistically significant correlation between the developmental responses to copper stress and adult copper resistance (Figure 4), we did observe evidence of partially shared genetic architectures between treatment-specific developmental viability and adult copper resistance (Figure 5B). Additional testing would be needed to determine whether the same genes implicated by developmental viability QTL Q15 and adult copper resistance QTL Q11 influence copper resistance at each life stage.

Because the ecology of the developing and adult stages of $D$. melanogaster are quite distinct, that copper resistance might be influenced by largely life stage-specific mechanisms is not unexpected. For example, D. melanogaster adults and larvae avoid copper-supplemented food when given the opportunity (Balamurugan et al. 2007; Bahadorani and Hilliker 2009); however, in natural populations, higher mobility of adults would allow the adult life stage to avoid heavy metal contaminated food more effectively. Life-stage specific genetic architectures were observed in D. melanogaster for cold tolerance (Freda et al. 2019), and the decoupling of the genetic mechanisms that influence survival and fitness have been reported in diverse organisms with complex life cycles (Moran 1994; Ragland and Kingsolver 2008). However, a number of other factors including the large difference in copper dose and the nature of the response tested at each life stage may obscure or complicate the relationship between the developmental and adult responses we observed. We used a much lower dose in our assessment of the effect of copper on development time and developmental viability compared to the adult copper resistance phenotype, and differences in dose can alter the genetic architecture for a trait. For example, in the yeast S. cerevisiae, Wang and Kruglyak (2014) demonstrated that the overall genetic architecture of haloperidol resistance was dosedependent. While one QTL was consistently detected for each of the five doses tested, several QTL were only detected at a single dose (Wang and Kruglyak 2014). With the added complexity of assessing the effects of copper in different life stages, it is difficult to fully determine whether the effects of copper in the adult and developmental assays are analogous. Further confounding this comparison, our adult copper assay was implemented over a 48$\mathrm{h}$ time period in contrast to exposing developing flies from egg to adult to copper over a period of 30 days at most. The harmful effects of copper on development may be constant or variable across different stages (egg, larvae, pupation), and this represents an area of ongoing research.

\section{Copper sensitivity is influenced by gene expression variation and behavior}

Differences in expression levels of genes that have protective functions against toxins, or that are co-opted by toxins can lead to variation in resistance levels. For instance in humans, natural variation in expression levels of the gene CMG2 is associated with variation in resistance to anthrax (Martchenko et al. 2012), and in the fungus Suillus luteus, selection pressure from heavy metal pollution quickly led to copy number variation in transport genes with protective functions against heavy metal toxicity (Bazzicalupo et al. 2019). Sensitivity to copper in adult D. melanogaster DSPR strains does not appear to be due to insufficient expression of genes involved with copper or metal detoxification such as MTs or CTR family transporters (Supplementary Table S5). Instead, we found that genes associated with metabolism and mitochondrial function were copper-induced in sensitive strains and copper-repressed in resistant strains (Figure 9). Stress caused by toxic levels of copper ions results in overproduction of ROS, which can alter energy production and metabolism (Uriu-Adams and Keen 2005; Tchounwou et al. 2008; Quijano et al. 2016). Negative effects of the misregulation of or overexposure to copper and other heavy metals such as zinc, lead, mercury, and arsenic through disease (Giacoppo et al. 2014; Liddell and White 2018; Umair and Alfadhel 2019) or because of pollution have been associated with impaired or altered mitochondrial function (Belyaeva et al. 2011, 2012; Jia et al. 2015). It is therefore possible that variation in metabolic function among the DSPR strains is one of the underlying contributors to variation in copper resistance. Given that we also observed that sensitive strains are slightly more likely to consume copper in larger amounts in a 24$\mathrm{h}$ period compared to resistant strains (Figure 2), sensitive strains may be under greater metabolic stress as they cope with exposure to behaviorally mediated higher levels of ingested copper. Copper resistance in D. melanogaster may not be simply a function of how well the organism is able to detoxify food; more likely, copper resistance is a combination of behavioral aversion to copper and the metabolic stress induced by the amount of metal consumed in addition to detoxification ability.

In general, food consumption rate has a complex genetic basis in D. melanogaster (Garlapow et al. 2015), and when given a choice, both D. melanogaster adults and larvae tend to avoid coppersupplemented food at much lower concentrations relative to those tested in this study (Balamurugan et al. 2007; Bahadorani and Hilliker 2009). Bahadorani and Hilliker (2009) showed that adult copper avoidance was observed at $1 \mathrm{mM} \mathrm{CuSO}_{4}$, and avoidance in third-instar larvae was observed at $0.25 \mathrm{mM} \mathrm{CuSO}_{4}$ (Balamurugan et al. 2007). Similarly, adult D. melanogaster avoid pupation and oviposition on copper-supplemented food (Bahadorani and Hilliker 2009). While this behavioral component likely plays an important role in mediating copper stress in natural populations, these studies focused on only one or few genetic strains, making it difficult to extrapolate how a genetically variable population would behave in response to copper. The correlation between adult copper resistance and copper food consumption in the 100 DSPR strains tested in our study suggests that variation in copper avoidance may play an important role in overall adult copper resistance. At this point, the specific relationship between copper consumption rates, metabolic stress, and genetic resistance to copper has not been characterized, but doing so in future studies has potential to more clearly define resistance to ingested toxins compared to an assessment based solely on survival. Important remaining questions include whether behavioral avoidance and sensitivity to heavy metals are influenced by variation in chemosensory detection ability (e.g., Arya et al. 2015; He et al. 2016) or variation in preference for metal-supplemented food (e.g., Highfill et al. 2019). Addressing these questions with a large panel such as the DSPR will help support our efforts to characterize the relationship and 
potential interaction between behavior and genetic capacity for copper resistance.

\section{Conclusions}

Copper resistance in D. melanogaster is genetically complex, is influenced by allelic and expression variation as well as by variation in behavioral avoidance of copper, and may be controlled by distinct sets of loci in different life stages. Several genes that have known copper-specific functions as well as genes that are involved in the regulation of other heavy metals were identified as potential candidates for variation in adult copper resistance and treatment-specific developmental viability. We demonstrated that nine of these candidates influenced adult copper resistance, providing evidence of pleiotropic effects of genes previously thought to be associated with other heavy metals. Copper is just one of many heavy metals that pollute the environment with negative impacts on humans, fungi, plants, and insects at a global scale. Understanding the complexity of the genetic basis of copper resistance and the potential sources of variation that interact with resistance is important for understanding the diverse mechanisms through which copper pollution can negatively impact organisms.

\section{Acknowledgments}

Stocks obtained from the Bloomington Drosophila Stock Center (NIH P40 OD018537) were used in this study. We thank Robert Unckless and John Kelly for their comments on the manuscript, and William Jewell College for use of the macro camera lens used in the developmental assays. We also thank three anonymous reviewers and Patricia Wittkopp for their suggestions that improved this manuscript.

\section{Funding}

ERE was supported by Postdoctoral Fellowships from the NIH (National Institutes of Health) (F32 GM133111) and the Kansas INBRE (Kansas Idea Network of Biomedical Research Excellence) project (P20 GM103418). The research was supported by NIH R01 OD010974 to S.J.M. and Anthony Long (UC Irvine), and by NIH R01 ES029922 to S.J.M., and computational work was supported by the Kansas INBRE (P20 GM103418). Sequencing was carried out by the KU Genome Sequencing Core which is supported by the CMADP (Center for Molecular Analysis of Disease Pathways), an NIH COBRE (Centers of Biomedical Research Excellence) award (P20 GM103638).

\section{Conflicts of interest}

None declared.

\section{Literature cited}

Abu-Jamous B, Kelly S. 2018. Clust: Automatic Extraction of Optimal Co-Expressed Gene Clusters from Gene Expression Data. doi: 10.1101/221309.

Allen WR. 1971. Copper tolerance in some Californian populations of the monkey flower, Mimulus guttatus. Proc R Soc Lond B Biol Sci. 177:177-196.

Arnold BJ, Lahner B, DaCosta JM, Weisman CM, Hollister JD, et al. 2016. Borrowed alleles and convergence in serpentine adaptation. Proc Natl Acad Sci U S A. 113:8320-8325. doi:10.1073/ pnas. 1600405113.

Arya GH, Magwire MM, Huang W, Serrano-Negron YL, Mackay TFC, et al. 2015. The genetic basis for variation in olfactory behavior in Drosophila melanogaster. Chem Senses. 40:233-243. doi:10.1093/ chemse/bjv001.

Babin-Fenske J, Anand M. 2011. Patterns of insect communities along a stress gradient following decommissioning of a $\mathrm{Cu}-\mathrm{Ni}$ smelter. Environ Pollut. 159:3036-3043. doi:10.1016/j.envpol. 2011.04.011.

Bahadorani S, Hilliker AJ. 2009. Biological and behavioral effects of heavy metals in Drosophila melanogaster adults and larvae. J Insect Behav. 22:399-411. doi:10.1007/s10905-009-9181-4.

Bahadorani S, Mukai S, Egli D, Hilliker AJ. 2010. Overexpression of metal-responsive transcription factor (MTF-1) in Drosophila melanogaster ameliorates life-span reductions associated with oxidative stress and metal toxicity. Neurobiol Aging. 31:1215-1226. doi:10.1016/j.neurobiolaging.2008.08.001.

Balamurugan K, Egli D, Hua H, Rajaram R, Seisenbacher G, et al. 2007. Copper homeostasis in Drosophila by complex interplay of import, storage and behavioral avoidance. EMBO J. 26:1035-1044. doi:10.1038/sj.emboj.7601543.

Balamurugan K, Egli D, Selvaraj A, Zhang B, Georgiev O, et al. 2004. Metal-responsive transcription factor (MTF-1) and heavy metal stress response in Drosophila and mammalian cells: a functional comparison. Biol Chem. 385: 597-603. doi:10.1515/BC.2004.074.

Bálint AF, Röder MS, Hell R, Galiba G, Börner A. 2007. Mapping of QTLs affecting copper tolerance and the $\mathrm{Cu}, \mathrm{Fe}, \mathrm{Mn}$ and $\mathrm{Zn}$ contents in the shoots of wheat seedlings. Biol Plant. 51:129-134. doi:10.1007/s10535-007-0025-9.

Bazzicalupo AL, Ruytinx J, Ke Y-H, Coninx L, Colpaert JV, et al. 2019. Incipient local adaptation in a fungus: evolution of heavy metal tolerance through allelic and copy-number variation. bioRxiv. doi:10.1101/832089.

Beavis WD, Grant D, Albertsen M, Fincher R. 1991. Quantitative trait loci for plant height in four maize populations and their associations with qualitative genetic loci. Theor Appl Genet. 83:141-145.

Bellion M, Courbot M, Jacob C, Guinet F, Blaudez D, et al. 2007. Metal induction of a Paxillus involutus metallothionein and its heterologous expression in Hebeloma cylindrosporum. New Phytol. 174: 151-158. doi:10.1111/j.1469-8137.2007.01973.x.

Belyaeva EA, Korotkov SM, Saris N-E. 2011. In vitro modulation of heavy metal-induced rat liver mitochondria dysfunction: a comparison of copper and mercury with cadmium. J Trace Elem Med Biol. 25:S63-S73. doi:10.1016/j.jtemb.2010.10.007.

Belyaeva EA, Sokolova TV, Emelyanova LV, Zakharova IO. 2012. Mitochondrial electron transport chain in heavy metal-induced neurotoxicity: effects of cadmium, mercury, and copper. Sci World J. 2012:1-14. doi:10.1100/2012/136063.

Blackney MJ, Cox R, Shepherd D, Parker JD. 2014. Cloning and expression analysis of Drosophila extracellular Cu Zn superoxide dismutase. Biosci Rep. 34:e00164.doi:10.1042/BSR20140133.

Boyle EA, Li YI, Pritchard JK. 2017. An expanded view of complex traits: from polygenic to omnigenic. Cell 169:1177-1186. doi:10. 1016/j.cell.2017.05.038

Bray NL, Pimentel H, Melsted P, Pachter L. 2016. Near-optimal probabilistic RNA-seq quantification. Nat Biotechnol. 34:525-527. doi:10.1038/nbt.3519.

Buchon N, Osman D, David FPA, Yu Fang H, Boquete J-P, et al. 2013. Morphological and molecular characterization of adult midgut compartmentalization in Drosophila. Cell Rep. 3:1725-1738., doi:10.1016/j.celrep.2013.04.001. 
Calap-Quintana P, González-Fernández J, Sebastiá-Ortega N, Llorens J, Moltó M. 2017. Drosophila melanogaster models of metal-related human diseases and metal toxicity. Int J Mol Sci. 18:1456.doi:10. 3390/ijms18071456.

Chen S, Zhou Y, Chen Y, Gu J. 2018. fastp: an ultra-fast all-in-one FASTQ preprocessor. bioRxiv. doi:10.1101/274100.

Cohen J. 1988. Statistica Power for the Behavioral Sciences. Hillsdale, NJ: Lawrence Erlbaum.

Collet J, Fellous S. 2019. Do traits separated by metamorphosis evolve independently? Concepts and methods. Proc R Soc B. 286: 20190445.doi:10.1098/rspb.2019.0445.

Courbot M, Willems G, Motte P, Arvidsson S, Roosens N, et al. 2007. A major quantitative trait locus for cadmium tolerance in Arabidopsis halleri colocalizes with HMA4, a gene encoding a heavy metal ATPase. Plant Physiol. 144:1052-1065. doi:10.1104/pp.106. 095133

Culotta VC, Klomp LWJ, Strain J, Casareno RLB, Krems B, et al. 1997. The copper chaperone for superoxide dismutase. J Biol Chem. 272:23469-23472. doi:10.1074/jbc.272.38.23469.

Danks DM. 1988. Copper deficiency in humans. Annu Rev Nutr. 8: 235-257. doi:10.1146/annurev.nu.08.070188.001315.

Domingo A, GonzáLez-Jurado JUAN, Maroto M, Diáz C, VinóS J, et al. 1998. Troponin-T is a calcium-binding protein in insect muscle: in vivo phosphorylation, muscle-specific isoforms and developmental profile in Drosophila melanogaster. J Muscle Res Cell Motil. 19:393-403.

Ecke F, Singh NJ, Arnemo JM, Bignert A, Helander B, et al. 2017. Sublethal lead exposure alters movement behavior in free-ranging golden eagles. Environ Sci Technol. 51:5729-5736. doi:10.1021/acs.est.6b06024.

Egli D, Domènech J, Selvaraj A, Balamurugan K, Hua H, et al. 2006b. The four members of the Drosophila metallothionein family exhibit distinct yet overlapping roles in heavy metal homeostasis and detoxification. Genes Cells. 11:647-658. doi:10.1111/j.13652443.2006.00971.x.

Egli D, Selvaraj A, Yepiskoposyan H, Zhang B, Hafen E, et al. 2003. Knockout of 'metal-responsive transcription factor' MTF-1 in Drosophila by homologous recombination reveals its central role in heavy metal homeostasis. EMBO J. 22:100-108. doi:10.1093/ emboj/cdg012.

Egli D, Yepiskoposyan H, Selvaraj A, Balamurugan K, Rajaram R, et al. 2006a. A family knockout of all four Drosophila metallothioneins reveals a central role in copper homeostasis and detoxification. Mol Cell Biol. 26:2286-2296. doi:10.1128/MCB.26.6.22862296.2006.

Ehrenreich IM, Torabi N, Jia Y, Kent J, Martis S, et al. 2010. Dissection of genetically complex traits with extremely large pools of yeast segregants. Nature 464:1039-1042. doi:10.1038/nature08923.

Evans KS, Brady SC, Bloom JS, Tanny RE, Cook DE, et al. 2018. Shared genomic regions underlie natural variation in diverse toxin responses. Genetics 210:1509-1525. doi:10.1534/genetics.118. 301311.

Everman ER, McNeil CL, Hackett JL, Bain CL, Macdonald SJ. 2019. Dissection of complex, fitness-related traits in multiple Drosophila mapping populations offers insight into the genetic control of stress resistance. Genetics 211:1449-1467. doi:10.1534/genetics. 119.301930.

Filshie BK, Poulson DF, Waterhouse DF. 1971. Ultrastructure of the copper accumulating region of the Drosophila larval midgut. Tissue Cell 3:77-102.

Freda PJ, Alex JT, Morgan TJ, Ragland GJ. 2017. Genetic decoupling of thermal hardiness across metamorphosis in Drosophila melanogaster. Integr Comp Biol. 57:999-1009. doi:10.1093/icb/icx102.
Freda PJ, Ali ZM, Heter N, Ragland GJ, Morgan TJ. 2019. Stage-specific genotype-by-environment interactions for cold and heat hardiness in Drosophila melanogaster. Heredity 123:479-491. doi:10. 1038/s41437-019-0236-9.

Gall JE, Boyd RS, Rajakaruna N. 2015. Transfer of heavy metals through terrestrial food webs: a review. Environ Monit Assess. 187: 1-21. doi:10.1007/s10661-015-4436-3.

Garlapow ME, Huang W, Yarboro MT, Peterson KR, Mackay TFC. 2015. Quantitative genetics of food Intake in Drosophila melanogaster. PLoS One 10: 1-25. doi:10.1371/journal.pone.0138129.

Gatti DM, Svenson KL, Shabalin A, Wu L-Y, Valdar W, et al. 2014. Quantitative trait locus mapping methods for diversity outbred mice. G3 (Bethesda) 4:1623-1633. doi:10.1534/g3.114.013748.

Gaudet P, Livstone M S, Lewis S E, Thomas P D. 2011. Phylogenetic-based propagation of functional annotations within the Gene Ontology consortium. Briefings in Bioinformatics. 12: 449-462. 10.1093/bib/bbr042

Georgieva SS, McGrath SP, Hooper DJ, Chambers BS. 2002. Nematode communities under stress: the long-term effects of heavy metals in soil treated with sewage sludge. Appl Soil Ecol. 20:27-42. doi:10.1016/S0929-1393(02)00005-7.

Giacoppo S, Galuppo M, Calabrò RS, D'Aleo G, Marra A, et al. 2014. Heavy metals and neurodegenerative diseases: an observational study. Biol Trace Elem Res. 161:151-160. doi:10.1007/s12011-0140094-5.

Gleason JM, Roy PR, Everman ER, Gleason TC, Morgan TJ. 2019. Phenology of Drosophila species across a temperate growing season and implications for behavior. PLoS One 14:e0216601.doi:10. 1371/journal.pone.0216601.

Goldsbrough P. 2000. Metal tolerance in plants: the role of phytochelatins and metallothioneins. Phytoremediation of Contaminated Soil and Water. Boca Raton, FL: CRC Press LLC. p. 227-239.

GTEx Consortium, Gamazon ER, Segrè AV, van de Bunt M, Wen X, Xi HS, et al. 2018. Using an atlas of gene regulation across 44 human tissues to inform complex disease- and trait-associated variation. Nat Genet. 50:956-967. doi:10.1038/s41588-018-0154-4.

Guo Y, Smith K, Lee J, Thiele DJ, Petris MJ. 2004. Identification of methionine-rich clusters that regulate copper-stimulated endocytosis of the human Ctr1 copper transporter. J Biol Chem. 279: 17428-17433. doi:10.1074/jbc.M401493200.

Guruharsha KG, Rual J-F, Zhai B, Mintseris J, Vaidya P, et al. 2011. A protein complex network of Drosophila melanogaster. Cell 147: 690-703. doi:10.1016/j.cell.2011.08.047.

Hart EB, Steenbock H, Waddell J, Elvehjeim CA. 1928. Iron nutrition. VII copper is a supplement to iron for hemoglobin building in rat. J Biol Chem. 77:797-812.

Harvey PJ, Handley HK, Taylor MP. 2016. Widespread copper and lead contamination of household drinking water, New South Wales, Australia. Environ Res. 151:275-285. doi:10.1016/j.envres. 2016.07.041.

Hatori Y, Lutsenko S. 2013. An expanding range of functions for the copper chaperone/antioxidant protein Atox1. Antioxid Redox Signal. 19:945-957. doi:10.1089/ars.2012.5086.

He X, Zhou S, St. Armour GE, Mackay TFC, Anholt RRH. 2016. Epistatic partners of neurogenic genes modulate Drosophila olfactory behavior: epistatic modifiers for Drosophila olfaction. Genes Brain Behav. 15:280-290. doi:10.1111/gbb.12279.

Highfill CA, Baker BM, Stevens SD, Anholt RRH, Mackay TFC. 2019. Genetics of cocaine and methamphetamine consumption and preference in Drosophila melanogaster. PLoS Genet. 15: e1007834.doi:10.1371/journal.pgen.1007834.

Highfill CA, Reeves GA, Macdonald SJ. 2016. Genetic analysis of variation in lifespan using a multiparental advanced intercross 
Drosophila mapping population. BMC Genet. 17: 1-13. doi:10.1186/ s12863-016-0419-9.

Huard DJE, Kane KM, Tezcan FA. 2013. Re-engineering protein interfaces yields copper-inducible ferritin cage assembly. Nat Chem Biol. 9:169-176. doi:10.1038/nchembio.1163.

Ilunga Kabeya F, Pongrac P, Lange B, Faucon M-P, van Elteren JT, et al. 2018. Tolerance and accumulation of cobalt in three species of Haumaniastrum and the influence of copper. Environ Exp Bot. 149: 27-33. doi:10.1016/j.envexpbot.2018.01.018.

Janssens TKS, Roelofs D, van Straalen NM. 2009. Molecular mechanisms of heavy metal tolerance and evolution in invertebrates. Insect Sci. 16:3-18. doi:10.1111/j.1744-7917.2009.00249.x.

Jia G, Aroor AR, Martinez-Lemus LA, Sowers JR. 2015. Mitochondrial functional impairment in response to environmental toxins in the cardiorenal metabolic syndrome. Arch Toxicol. 89:147-153. doi:10.1007/s00204-014-1431-3.

Keller A. 2007. Drosophila melanogaster's history as a human commensal. Curr Biol. 17:R77-R81. doi:10.1016/j.cub.2006.12.031.

King EG, Macdonald SJ, Long AD. 2012a. Properties and power of the Drosophila synthetic population resource for the routine dissection of complex traits. Genetics 191:935-949. doi:10.1534/genet ics.112.138537.

King EG, Merkes CM, McNeil CL, Hoofer SR, Sen S, et al. 2012b. Genetic dissection of a model complex trait using the Drosophila synthetic population resource. Genome Res. 22:1558-1566. doi:10.1101/gr.134031.111.

King EG, Kislukhin G, Walters KN, Long AD. 2014. Using Drosophila melanogaster to identify chemotherapy toxicity genes. Genetics 198:31-43. doi:10.1534/genetics.114.161968.

King EG, Long AD. 2017. The Beavis effect in next-generation mapping panels in Drosophila melanogaster. G3 (Bethesda) 7:1643-1652.

Kirby K, Jensen LT, Binnington J, Hilliker AJ, Ulloa J, et al. 2008. Instability of superoxide dismutase 1 of Drosophila in mutants deficient for its cognate copper chaperone. J Biol Chem. 283: 35393-35401. doi:10.1074/jbc.M807131200.

Kislukhin G, King EG, Walters KN, Macdonald SJ, Long AD. 2013. The genetic architecture of methotrexate toxicity is similar in Drosophila melanogaster and humans. G3 (Bethesda) 3:1301-1310. doi:10.1534/g3.113.006619.

Liddell JR, White AR. 2018. Nexus between mitochondrial function, iron, copper and glutathione in Parkinson's disease. Neurochem Int. 117:126-138. doi:10.1016/j.neuint.2017.05.016.

Lüdecke D. 2018. sjstats: Statistical Functions for Regression Models. Version 0.18.0. doi: 10.5281/zenodo.1284472, https://CRAN.R-proj ect.org/package $=$ sjstats

Lye JC, Richards CD, Dechen K, Warr CG, Burke R. 2013. In vivo zinc toxicity phenotypes provide a sensitized background that suggests zinc transport activities for most of the Drosophila Zip and ZnT genes. J Biol Inorg Chem. 18:323-332. doi:10.1007/s00775013-0976-6.

Lynch M, Walsh B. 1998. Genetic and Analysis of Quantitative Traits. Sunderland, MA: Sinauer.

Lyne R, Smith R, Rutherford K, Wakeling M, Varley A, et al. 2007. FlyMine: an integrated database for Drosophila and Anopheles genomics. Genome Biol. 8:R129. doi:10.1186/gb-2007-8-7-r129.

Mackay TFC, Richards S, Stone EA, Barbadilla A, Ayroles JF, et al. 2012. The Drosophila melanogaster genetic reference panel. Nature 482:173-178. doi:10.1038/nature10811.

Macnair MR. 1983. The genetic control of copper tolerance in the yellow monkey flower, Mimulus guttatus. Heredity 50:283-293.

Macnair MR. 1993. The genetics of metal tolerance in vascular plants. New Phytol. 124:541-559. doi:10.1111/j.1469-8137.1993. tb03846.x.
MacNair MR, Smith SE, Cumbes QJ. 1993. Heritability and distribution of variation in degree of copper tolerance in Mimulus guttatus at Copperopolis, California. Heredity 71:445-455. doi:10.1038/ hdy.1993.162.

Maroni G, Watson D. 1985. Uptake and binding of cadmium, copper and zinc by Drosophila melanogaster larvae. Insect Biochem. 15: 55-63.

Marriage TN, King EG, Long AD, Macdonald SJ. 2014. Fine-mapping nicotine resistance loci in Drosophila using a multiparent advanced generation inter-cross population. Genetics 198:45-57. doi:10.1534/genetics.114.162107

Martchenko M, Candille SI, Tang H, Cohen SN. 2012. Human genetic variation altering anthrax toxin sensitivity. Proc Natl Acad Sci U S A. 109:2972-2977. doi:10.1073/pnas.1121006109.

Mercer SW, Burke R. 2016. Evidence for a role for the putative Drosophila hGRX1 orthologue in copper homeostasis. Biometals 29:705-713. doi:10.1007/s10534-016-9946-0.

Mercer SW, Fontaine SL, Warr CG, Burke R. 2016. Reduced glutathione biosynthesis in Drosophila melanogaster causes neuronal defects linked to copper deficiency. J Neurochem. 137:360-370. doi:10.1111/jnc.13567.

Mokdad R, Debec A, Wegnez M. 1987. Metallothionein genes in Drosophila melanogaster constitute a dual system. Proc Natl Acad Sci U S A. 84:2658-2662.

Montgomery SL, Vorojeikina D, Huang W, Mackay TFC, Anholt RRH, et al. 2014. Genome-wide association analysis of tolerance to methylmercury toxicity in Drosophila implicates myogenic and neuromuscular developmental pathways. PLoS One 9:e110375.

Moran NA. 1994. Adaptation and constraint in the complex Life cycles of Animals. Annu Rev Ecol Syst. 25:573-600.

Najarro MA, Hackett JL, Macdonald SJ. 2017. Loci contributing to boric acid toxicity in two reference populations of Drosophila melanogaster. G3 (Bethesda) 7:1631-1641.

Navarro JA, Schneuwly S. 2017. Copper and zinc homeostasis: lessons from Drosophila melanogaster. Front Genet. 8:1-20. doi:10. 3389/fgene.2017.00223.

Neuberger JS, Mulhall M, Pomatto MC, Sheverbush J, Hassanein RS. 1990. Health problems in Galena, Kansas: a heavy metal mining Superfund site. Sci Total Environ. 94:261-272.

Ni J-Q, Liu L-P, Binari R, Hardy R, Shim H-S, et al. 2009. A Drosophila resource of transgenic RNAi lines for neurogenetics. Genetics 182: 1089-1100. doi:10.1534/genetics.109.103630.

Ni J-Q, Zhou R, Czech B, Liu L-P, Holderbaum L, et al. 2011. A genome-scale shRNA resource for transgenic RNAi in Drosophila. Nat Methods. 8:405-407. doi:10.1038/nmeth.1592.

Norgate M, Lee E, Southon A, Farlow A, Batterham P, et al. 2006. Essential roles in development and pigmentation for the Drosophila copper transporter DmATP7. Mol Biol Cell. 17:475-484. doi:10.1091/mbc.E05-06-0492.

Norgate M, Southon A, Greenough M, Cater M, Farlow A, et al. 2010. Syntaxin 5 is required for copper homeostasis in Drosophila and mammals. PLoS One 5:e14303. doi:10.1371/journal.pone.0014303.

Norgate M, Southon A, Zou S, Zhan M, Sun Y, et al. 2007. Copper homeostasis gene discovery in Drosophila melanogaster. Biometals 20: 683-697. doi:10.1007/s10534-006-9075-2.

Oliveira-Filho E. C D, Lopes RM, Paumgartten FJR. 2004. Comparative study on the susceptibility of freshwater species to copper-based pesticides. Chemosphere 56:369-374. doi:10.1016/j.chemosphere. 2004.04.026.

Ozdowski EF, Mowery YM, Cronmiller C. 2009. stall encodes an ADAMTS metalloprotease and interacts genetically with Delta in Drosophila ovarian follicle formation. Genetics 183:1027-1040. doi:10.1534/genetics.109.107367. 
Paradis E, Claude J, Strimmer K. 2004. APE: analysis of phylogenetics and evolution in R language. Bioinformatics 20:289-290.

Perkins LA, Holderbaum L, Tao R, Hu Y, Sopko R, et al. 2015. The transgenic RNAi project at Harvard Medical School: resources and validation. Genetics 201:843-852. doi:10.1534/genetics.115. 180208

Petris MJ, Smith K, Lee J, Thiele DJ. 2003. Copper-stimulated endocytosis and degradation of the human copper transporter, hCtr1. J Biol Chem. 278:9639-9646. doi:10.1074/jbc.M209455200.

Pimentel H, Bray NL, Puente S, Melsted P, Pachter L. 2017. Differential analysis of RNA-seq incorporating quantification uncertainty. Nat Methods. 14:687-690. doi:10.1038/nmeth.4324.

Pinheiro J, Bates D, DebRoy S, Sarkar D, Team RC. 2017. nlme: linear and nonlinear mixed effects models. Version 3.1-151. https:// CRAN.R-project.org/package $=$ nlme.

Plessl C, Jandrisits P, Krachler R, Keppler BK, Jirsa F. 2017. Heavy metals in the mallard Anas platyrhynchos from eastern Austria. Sci Total Environ. 580:670-676. doi:10.1016/j.scitotenv.2016.12.013.

Pölkki M. 2016. The effects of copper exposure on life-history traits in insects. Ann Univ Turku. 328: 1-44.

Quijano C, Trujillo M, Castro L, Trostchansky A. 2016. Interplay between oxidant species and energy metabolism. Redox Biol. 8: 28-42. doi:10.1016/j.redox.2015.11.010.

R Core Team. 2017. R: A Language and Environment for Statistical Computing. Vienna, Austria: R Foundation for Statistical Computing.

Ragland GJ, Kingsolver JG. 2008. Evolution of thermotolerance in seasonal environments: the effects of annual temperature variation and life-history timing in Wyeomyia smithii. Evolution 62: 1345-1357. doi:10.1111/j.1558-5646.2008.00367.x.

Ramirez M, Massolo S, Frache R, Correa JA. 2005. Metal speciation and environmental impact on sandy beaches due to El Salvador copper mine, Chile. Mar Pollut Bull. 50:62-72. doi:10.1016/j.mar polbul.2004.08.010.

Roelofs D, Overhein L, de Boer ME, Janssens TKS, van Straalen NM. 2006. Additive genetic variation of transcriptional regulation: metallothionein expression in the soil insect Orchesella cincta. Heredity 96:85-92. doi:10.1038/sj.hdy.6800756.

Ruden DM, Chen L, Possidente D, Possidente B, Rasouli P, et al. 2009. Genetical toxicogenomics in Drosophila identifies master-modulatory loci that are regulated by developmental exposure to lead. NeuroToxicology 30:898-914. doi:10.1016/j.neuro. 2009.08.011.

Ryabinina O, Subbian E, Iordanov M. 2006. D-MEKKI, the Drosophila orthologue of mammalian MEKK4/MTKI, and Hemipterous/D-MKK7 mediate the activation of D-JNK by cadmurm and arsenite in Schneider cells. BMC Cell Biol. 7:7.doi: 10.1186/1471-2121-7-7.

Sánchez-Chardi A, Nadal J. 2007. Bioaccumulation of metals and effects of landfill pollution in small mammals. Part I. The greater white-toothed shrew, Crocidura russula. Chemosphere 68:703-711. doi:10.1016/j.chemosphere.2007.01.042.

Schmidt PJ, Kunst C, Culotta VC. 2000. Copper activation of superoxide dismutase 1 (SOD1) in vivo: role for protein-protein interactions with the copper chaperone for SOD1. J Biol Chem. 275: 33771-33776. doi:10.1074/jbc.M006254200.

Selby JP, Willis JH. 2018. Major QTL controls adaptation to serpentine soils in Mimulus guttatus. Mol Ecol. 27:5073-5087. doi:10.1111/ mec.14922.

Shell BC, Schmitt RE, Lee KM, Johnson JC, Chung BY, et al. 2018. Measurement of solid food intake in Drosophila via consumption-excretion of a dye tracer. Sci Rep. 8. doi: 10.1038/s41598-018-29813-9.
Southon A, Burke R, Norgate M, Batterham P, Camakaris J. 2004. Copper homoeostasis in Drosophila melanogaster S2 cells. Biochem J. 383:303-309. doi:10.1042/BJ20040745.

Stern DL. 2014. Identification of loci that cause phenotypic variation in diverse species with the reciprocal hemizygosity test. Trends Genet. 30:547-554. doi:10.1016/j.tig.2014.09.006.

Stuart GW, Searle PF, Palmiter RD. 1985. Identification of multiple metal regulatory elements in mouse metallothionein-I promoter by assaying synthetic sequences. Nature 317:828-831. doi:10. 1038/317828a0.

Tchounwou PB, Newsome C, Williams J, Glass K. 2008. Copper-induced cytotoxicity and transcriptional activation of stress genes in human liver carcinoma (HepG2) cells. Met Ions Biol Med. 10:285-290.

Terhzaz S, Cabrero P, Chintapalli VR, Davies S-A, Dow JAT. 2010. Mislocalization of mitochondria and compromised renal function and oxidative stress resistance in Drosophila SesB mutants. Physiol Genomics. 41:33-41. doi:10.1152/physiolgenomics.00147. 2009.

Thurmond J, Goodman JL, Strelets VB, Attrill H, Gramates LS, et al. 2019. FlyBase 2.0: the next generation. Nucleic Acids Res. 47: D759-D765. doi:10.1093/nar/gky1003.

Turner TL, Bourne EC, Von Wettberg EJ, Hu TT, Nuzhdin SV. 2010. Population resequencing reveals local adaptation of Arabidopsis lyrata to serpentine soils. Nat Genet. 42:260-263. doi:10.1038/ng. 515.

Turski ML, Thiele DJ. 2007. Drosophila Ctr1A functions as a copper transporter essential for development. J Biol Chem. 282: 24017-24026. doi:10.1074/jbc.M703792200.

Umair M, Alfadhel M. 2019. Genetic disorders associated with metal metabolism. Cells 8:1598.doi:10.3390/cells8121598.

Uriu-Adams JY, Keen CL. 2005. Copper, oxidative stress, and human health. Mol Aspects Med. 26:268-298. doi:10.1016/j.mam.2005.07. 015.

Usmani JM. 2011. Accumulation of heavy metals in fishes: a human health concern. Int J Environ Sci. 2:671-682.

Wang X, Kruglyak L. 2014. Genetic basis of haloperidol resistance in Saccharomyces cerevisiae is complex and dose dependent. PLoS Genet. 10:e1004894. doi:10.1371/journal.pgen.1004894.

Willems G, Dräger DB, Courbot M, Godé C, Verbruggen N, et al. 2007. The genetic basis of zinc tolerance in the metallophyte Arabidopsis halleri ssp. halleri (Brassicaceae): an analysis of quantitative trait loci. Genetics 176:659-674. doi:10.1534/genetics.106. 064485 .

World Health Organization, Food and Agriculture Organization of the United Nations, and International Atomic Energy Agency. 1996. Trace Elements in Human Nutrition and Health. Geneva: World Health Organization.

Wright KM, Hellsten U, Xu C, Jeong AL, Sreedasyam A, et al. 2015. Adaptation to heavy-metal contaminated environments proceeds via selection on pre-existing genetic variation. bioRxiv. doi:10.1101/029900.

Wu L, Bradshaw AD, Thurman DA. 1975. The potential for evolution of heavy metal tolerance in plants. III. The rapid evolution of copper tolerance in Agrostis stolonifera. Heredity 34:165-187.

Wuana RA, Okieimen FE. 2011. Heavy metals in contaminated soils: a review of sources, chemistry, risks and best available strategies for remediation. ISRN Ecol. 2011:1-20. doi:10.5402/2011/402647.

Yepiskoposyan H, Egli D, Fergestad T, Selvaraj A, Treiber C, et al. 2006. Transcriptome response to heavy metal stress in Drosophila reveals a new zinc transporter that confers resistance to zinc. Nucleic Acids Res. 34:4866-4877. doi:10.1093/nar/ gkl606. 
Yin S, Qin Q, Zhou B. 2017. Functional studies of Drosophila zinc transporters reveal the mechanism for zinc excretion in Malpighian tubules. BMC Biol. 15:12.doi:10.1186/s12915-017-0355-9.

Zhou H, Cadigan KM, Thiele DJ. 2003. A copper-regulated transporter required for copper acquisition, pigmentation, and specific stages of development in Drosophila melanogaster. J Biol Chem. 278:48210-48218. doi:10.1074/jbc.M309820200.

Zhou S, Luoma SE, St. Armour GE, Thakkar E, Mackay TFC, et al. 2017. A Drosophila model for toxicogenomics: genetic variation in susceptibility to heavy metal exposure. PLoS Genet. 13:e1006907. doi:10.1371/journal.pgen.1006907.

Zhou S, Morozova TV, Hussain YN, Luoma SE, McCoy L, et al. 2016.

The genetic basis for variation in sensitivity to lead toxicity in Drosophila melanogaster. Environ Health Perspect. 124:1062-1070. doi:10.1289/ehp.1510513.

Communicating editor: P. Wittkopp 